\title{
Prioritized Inverse Kinematics: Generalization
}

\author{
Sang-ik $\mathrm{An}^{1}$, Student Member, IEEE, and Dongheui Lee ${ }^{1,2}$, Senior Member, IEEE,
}

\begin{abstract}
This letter proposes a generalization of the prioritized inverse kinematics (PIK) problem as the multi-objective optimization with the lexicographical ordering. We specify three properties for a vector-valued objective function to be proper for the PIK problem, so that the set of all PIK solutions can be generated from the set of all proper objective functions. The dependence property requires that higher priority tasks do not constrain lower priority tasks unnecessarily, the uniqueness property demands that there exists one and only one PIK solution given a proper objective function, and the representation property asks that if there are two distinct references that are realizable by a mechanism, then the PIK solutions for those references should differ. We also include the preconditioning of the velocity mapping functions in our generalization that can be used to handle the numerical imbalance in the orthogonalization that raises a difficulty in choosing damping functions and degenerates the performance of lower priority tasks. We justify our generalization by showing that it discards trivial solutions such as a constant function that is not intended and contains several PIK solutions including two successful PIK solutions, so called Nakamura's and (weighted) Chiaverini's solutions, with and without damping. We compare those PIK solutions by a simulation with a seven degrees of freedom manipulator, KUKALWR.
\end{abstract}

Index Terms-Kinematics, redundant robots, optimization and optimal control, prioritized inverse kinematics.

\section{INTRODUCTION}

$\mathbf{P}$ RIORITIZED Inverse Kinematics (PIK) in the velocity level has been studied intensively for decades along with the growth of the robotic society and the study on the PIK has been used and expanded in many areas such as constrained PIK [1][2][3][4], task switching [5][6][7][8][9], prioritized control [10][11][12][13][14][15], prioritized optimal control [16][17][18][19][20], learning prioritized tasks [21][22][23][24][25][26], etc. Nowadays, the PIK and the related studies are considered as a basic building block in the development of learning and control methods for robotic systems that have multiple tasks to accomplish. In spite of the enormous studies on the PIK, most of them are focused on the formulation and the practical use of a specific PIK solution on their own necessity. However, there are some questions that those approaches cannot answer. For example, can we

Manuscript received: February, 28, 2019; Revised May, 30, 2019; Accepted June, 25, 2019.

This paper was recommended for publication by Editor Dezhen Song upon evaluation of the Associate Editor and Reviewers' comments. This work was supported in part by Technical University of Munich - Institute for Advanced Study, funded by the German Excellence Initiative. (Corresponding Author: Dongheui Lee.)

${ }^{1}$ The authors are with the Human-Centered Assistive Robotics, Department of Electrical and Computer Engineering, Technical University of Munich, D80333 Munich, Germany. \{sangik.an, dhlee\}@tum.de

${ }^{2} \mathrm{D}$. Lee is also with Institute of Robotics and Mechatronics, German Aerospace Center (DLR).

Digital Object Identifier (DOI): see top of this page. always find a smooth PIK solution given smooth Jacobian matrices and smooth references? Smoothness of a PIK solution is an important property we must check because existence and uniqueness of a joint trajectory satisfying a PIK solution depend on it. To answer this question, we need to know the set of all PIK solutions. However, based on our knowledge, no one defined such a set.

In this letter, we propose a generalization of the PIK problem. In Section II, we provide a background of the PIK problem focusing on two successful PIK solutions. The idea to see a specific PIK solution as an optimal solution of the multi-objective optimization with the lexicographical ordering can be found in [4][27]. In Section III, we generalize this idea by specifying three properties for a vector-valued objective function to be proper for the PIK problem and by preconditioning the velocity mapping functions. In our generalization, the set of all PIK solutions can be generated from the set of all proper objective functions by the mapping defined as the optimal solution of the multi-objective optimization with the lexicographical ordering. In Section IV, we show that our generalization discards trivial solutions such as a constant function that is not intended and contains several PIK solutions including two successful PIK solutions with and without damping. We also provide simulation results that compare those PIK solutions with a seven degrees of freedom (DOF) manipulator, KUKA LWR. The concluding remarks are followed in Section V.

\section{BACKGROUND}

Let $l \in \mathbb{N} \backslash\{1\}$ be the number of tasks. The forward kinematics with multiple tasks in the velocity level is a process to find the matrix-valued functions $\mathbf{J}_{a}: \mathbb{R}^{n} \rightarrow \mathbb{R}^{m_{a} \times n}$ for $a \in\{1, \ldots, l\}$ called the velocity mapping functions whose value $\mathbf{J}_{a}(\mathbf{q})$ maps the joint velocity $\dot{\mathbf{q}} \in \mathbb{R}^{n}$ into the $a$-th task velocity $\mathbf{J}_{a}(\mathbf{q}) \dot{\mathbf{q}} \in \mathbb{R}^{m_{a}}$ for each joint position $\mathbf{q} \in \mathbb{R}^{n}$. Without loss of generality, we assume $m=m_{1}+\cdots+m_{l} \leq n$. Here, the expression 'without loss of generality' means that we can always define the velocity mapping functions to satisfy this assumption. For example, if velocity mapping functions $\tilde{\mathbf{J}}_{a}: \mathbb{R}^{\tilde{n}} \rightarrow \mathbb{R}^{m_{a}}$ satisfy $m>\tilde{n}$, then we can redefine $\tilde{\mathbf{J}}_{a}$ to $\mathbf{J}_{a}: \mathbb{R}^{n} \rightarrow \mathbb{R}^{m_{a}}$ as

$$
\mathbf{J}_{a}(\mathbf{q})=\mathbf{J}_{a}\left(\tilde{\mathbf{q}}, q_{\tilde{n}+1}, \ldots, q_{n}\right)=\left[\tilde{\mathbf{J}}_{a}(\tilde{\mathbf{q}}) \quad \mathbf{0}\right]
$$

such that $m=n$ and $\mathbf{J}(\mathbf{q}) \dot{\mathbf{q}}=\tilde{\mathbf{J}}(\tilde{\mathbf{q}}) \dot{\tilde{\mathbf{q}}}$ by introducing dummy variables or virtual joints $q_{\tilde{n}+1}, \ldots, q_{n}$. Sometimes, $\mathbf{J}_{a}$ also depends on the time $t \in \mathbb{R}$ (e.g. the relative velocity between the end-effector and a moving object) but we restrict ourselves to the case $\mathbf{J}_{a}(\mathbf{q})$ at the moment. We may or may not require that $\mathbf{J}_{a}$ be the derivative of a function. For example, $\mathbf{J}_{a}(\mathbf{q}) \dot{\mathbf{q}}$ could be the angular velocity or the derivative of the Euler 
angles of a specific part of a mechanism. The desired behavior of the $a$-th task velocity $\mathbf{J}_{a}(\mathbf{q}) \dot{\mathbf{q}}$ is represented by the vector field $\mathbf{r}_{a}: \mathbb{R} \times \mathbb{R}^{n} \rightarrow \mathbb{R}^{m_{a}}$ called the $a$-th reference. The references are designed freely depending on a scenario of a mechanism in an environment. A typical choice could be $\mathbf{r}_{a}(t, \mathbf{q})=\dot{\mathbf{p}}_{a}(t)+\mathbf{K}_{a}\left(\mathbf{p}_{a}(t)-\mathbf{f}_{a}(\mathbf{q})\right)$ if $\mathbf{J}_{a}$ is the derivative of a forward kinematic function $\mathbf{f}_{a}: \mathbb{R}^{n} \rightarrow \mathbb{R}^{m_{a}}$ and the task position $\mathbf{f}_{a}(\mathbf{q})$ needs to follow a desired task trajectory $\mathbf{p}_{a}$ : $\mathbb{R} \rightarrow \mathbb{R}^{m_{a}}$ where $\mathbf{K}_{a} \in \mathbb{R}^{m_{a} \times m_{a}}$ is a feedback gain matrix. The goal of the $a$-th task at $(t, \mathbf{q})$ is to find the joint velocity $\dot{\mathbf{q}}$ that satisfies the $a$-th differential forward kinematics equation $\mathbf{J}_{a}(\mathbf{q}) \dot{\mathbf{q}}=\mathbf{r}_{a}(t, \mathbf{q})$ at least approximately or equivalently that minimizes the $a$-th residual $\mathbf{e}_{a}^{\text {res }}(t, \mathbf{q}, \mathbf{y})=\mathbf{r}_{a}(t, \mathbf{q})-\mathbf{J}_{a}(\mathbf{q}) \mathbf{y}$ with respect to $\mathbf{y}$ in some sense. The inverse kinematics with multiple tasks in the velocity level is a process to find the inverse kinematics solution $\mathbf{u}: \mathbb{R} \times \mathbb{R}^{n} \rightarrow \mathbb{R}^{n}$ satisfying

$$
\mathbf{J}(\mathbf{q}) \mathbf{u}(t, \mathbf{q})=\mathbf{r}(t, \mathbf{q})
$$

for each $(t, \mathbf{q}) \in \mathbb{R} \times \mathbb{R}^{n}$ at least approximately where $\mathbf{J}=\left[\begin{array}{lll}\mathbf{J}_{1}^{T} & \cdots & \mathbf{J}_{l}^{T}\end{array}\right]^{T}: \mathbb{R}^{n} \rightarrow \mathbb{R}^{m \times n}$ and $\mathbf{r}=\left(\mathbf{r}_{1}, \ldots, \mathbf{r}_{l}\right):$ $\mathbb{R}^{n} \rightarrow \mathbb{R}^{m}$. If such a function $\mathbf{u}$ is found, then the $a$-th residual can be written as $\mathbf{e}_{a}^{\text {res }}(t, \mathbf{q})=\mathbf{r}_{a}(t, \mathbf{q})-\mathbf{J}_{a}(\mathbf{q}) \mathbf{u}(t, \mathbf{q})$ and be used for the quantitative evaluation of $\mathbf{u}$.

In the PIK problem, the available DOF of a mechanism is the limited common resource that should be distributed to multiple tasks. Specifically, $\operatorname{rank}\left(\mathbf{J}_{a}(\mathbf{q})\right)$ is the maximum available DOF for the $a$-th task at $\mathbf{q} \in \mathbb{R}^{n}$. Here, $\operatorname{rank}(\cdot)$ is the rank of a matrix. In total, there $\operatorname{are} \operatorname{rank}(\mathbf{J}(\mathbf{q}))$ available DOF for all tasks and $\operatorname{rank}(\mathbf{J}(\mathbf{q})) \leq \sum_{a=1}^{l} \operatorname{rank}\left(\mathbf{J}_{a}(\mathbf{q})\right) \leq m$ by singularity. The $a$-th task is said to have a task singularity at $\mathbf{q}$ if $\operatorname{rank}\left(\mathbf{J}_{a}(\mathbf{q})\right)<m_{a}$. The $a$-th and $b$-th tasks are said to have

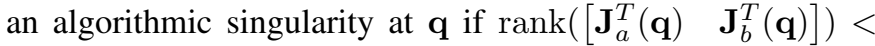
$\operatorname{rank}\left(\mathbf{J}_{a}(\mathbf{q})\right)+\operatorname{rank}\left(\mathbf{J}_{b}(\mathbf{q})\right)$. If the $a$-th task does not have a task singularity at $\mathbf{q}$, then we can always find $\dot{\mathbf{q}} \in \mathbb{R}^{n}$ satisfying $\mathbf{J}_{a}(\mathbf{q}) \dot{\mathbf{q}}=\mathbf{r}_{a}(t, \mathbf{q})$. However, even though the $a$-th and $b$-th tasks do not have task singularities at $\mathbf{q}$, if they have an algorithmic singularity, then we cannot always find $\dot{\mathbf{q}}$ satisfying $\mathbf{J}_{a}(\mathbf{q}) \dot{\mathbf{q}}=\mathbf{r}_{a}(t, \mathbf{q})$ and $\mathbf{J}_{b}(\mathbf{q}) \dot{\mathbf{q}}=\mathbf{r}_{b}(t, \mathbf{q})$ simultaneously. Priority is a strategy to distribute the available DOF to multiple tasks and the distribution is carried out by the consecutive projections of the joint velocity to the null spaces of the higher priority tasks.

There are two successful PIK solutions. The first solution, which we call the Nakamura's solution, was formulated by Nakamura et al. [28] and Maciejewski and Klein [29] for two tasks and extended later for an arbitrary number of tasks by Siciliano and Slotine [30] as

$$
\begin{aligned}
\mathbf{u}_{a} & =\mathbf{u}_{a-1}+\left(\mathbf{J}_{a} \mathbf{N}_{a-1}\right)^{\times}\left(\mathbf{r}_{a}-\mathbf{J}_{a} \mathbf{u}_{a-1}\right) \\
\mathbf{N}_{a} & =\mathbf{N}_{a-1}-\left(\mathbf{J}_{a} \mathbf{N}_{a-1}\right)^{+}\left(\mathbf{J}_{a} \mathbf{N}_{a-1}\right)
\end{aligned}
$$

where $\mathbf{u}=\mathbf{u}_{l}, \mathbf{u}_{0}=\mathbf{0}, \mathbf{N}_{0}=\mathbf{I}_{n},\left(\mathbf{J}_{a} \mathbf{N}_{a-1}\right)^{\times}=\left(\mathbf{J}_{a} \mathbf{N}_{a-1}\right)^{+}$ for $\lambda_{a}=0$, and $\left(\mathbf{J}_{a} \mathbf{N}_{a-1}\right)^{\times}=\left(\mathbf{J}_{a} \mathbf{N}_{a-1}\right)^{*}$ for $\lambda_{a} \in(0, \infty)$. Here, $\mathbf{I}_{n} \in \mathbb{R}^{n \times n}$ is the identity matrix, $\left(\mathbf{J}_{a} \mathbf{N}_{a-1}\right)^{+}$is the (Moore-Penrose) pseudoinerse, and $\left(\mathbf{J}_{a} \mathbf{N}_{a-1}\right)^{*}$ is the damped pseudoinverse with the damping constant $\lambda_{a}$ defined as $\left(\mathbf{J}_{a} \mathbf{N}_{a-1}\right)^{*}=\left(\mathbf{J}_{a} \mathbf{N}_{a-1}\right)^{T}\left(\mathbf{J}_{a} \mathbf{N}_{a-1} \mathbf{J}_{a}^{T}+\lambda_{a}^{2} \mathbf{I}_{m_{a}}\right)^{-1}$. In the early history of the prioritized inverse kinematics, there was not a fast and reliable way to compute the pseudoinverse for real-time applications, so the damped pseudoinverse $\left(\mathbf{J} \mathbf{N}_{a-1}\right)^{*}$ was often used in (2) instead of $\left(\mathbf{J}_{a} \mathbf{N}_{a-1}\right)^{+}$. However, this practical modification makes the projection imperfect and $\left(\mathbf{J}_{a} \mathbf{N}_{a-1}\right)^{*}$ in (1) used to cause unexpected behavior of the joint trajectory generated from the Nakamura's solution especially in the vicinity of the algorithmic singularities. It motivated researchers to think of a new prioritized inverse kinematics solution that is robust against algorithmic singularities.

The second solution, which we call the Chiaverini's solution, was proposed by Chiaverini [31] for two tasks and extended later for an arbitrary number of tasks by Baerlocher and Boulic [32] as

$$
\begin{aligned}
\mathbf{u}_{a} & =\mathbf{u}_{a-1}+\mathbf{N}_{a-1} \mathbf{J}_{a}^{\times} \mathbf{r}_{a} \\
\mathbf{N}_{a} & =\mathbf{N}_{a-1}-\left(\mathbf{J}_{a} \mathbf{N}_{a-1}\right)^{+}\left(\mathbf{J}_{a} \mathbf{N}_{a-1}\right)
\end{aligned}
$$

where $\mathbf{u}=\mathbf{u}_{l}, \mathbf{u}_{0}=\mathbf{0}, \mathbf{N}_{0}=\mathbf{I}_{n}, \mathbf{J}_{a}^{\times}=\mathbf{J}_{a}^{+}$for $\lambda_{a}=0$, and $\mathbf{J}_{a}^{\times}=\mathbf{J}_{a}^{*}$ for $\lambda_{a} \in(0, \infty)$. The Chiaverini's solution is known to be more robust against the algorithmic singularity but to generate the larger residual compared to the Nakamura's solution. To alleviate this inaccuracy problem of the second solution, Park et al. [33] and Choi et al. [34] modified the Chiaverini's solution using the weighted pseudoinverse.

Let $\delta \in(0, \infty)$ and define $\mathbf{W}: \mathbb{R}^{n} \rightarrow \mathbb{R}^{n \times n}$ as $\mathbf{W}(\mathbf{q})=$ $\mathbf{J}^{T}(\mathbf{q}) \mathbf{J}(\mathbf{q})+\delta^{2} \mathbf{I}_{n}$. The weighted Chiaverini's solution is given as

$$
\begin{aligned}
\mathbf{u}_{a} & =\mathbf{u}_{a-1}+\mathbf{N}_{a-1}^{W} \mathbf{J}_{a}^{W \times} \mathbf{r}_{a} \\
\mathbf{N}_{a}^{W} & =\mathbf{N}_{a-1}^{W}-\left(\mathbf{J}_{a} \mathbf{N}_{a-1}^{W}\right)^{W+}\left(\mathbf{J}_{a} \mathbf{N}_{a-1}^{W}\right)
\end{aligned}
$$

where $\mathbf{u}=\mathbf{u}_{l}, \mathbf{u}_{0}=\mathbf{0}, \mathbf{N}_{0}^{W}=\mathbf{I}_{n}, \mathbf{J}_{a}^{W \times}=\mathbf{J}_{a}^{W+}$ for $\lambda_{a}=0$, and $\mathbf{J}_{a}^{W \times}=\mathbf{J}_{a}^{W *}$ for $\lambda_{a} \in(0, \infty)$. Here, $\mathbf{J}_{a}^{W+}$ and $\left(\mathbf{J}_{a} \mathbf{N}_{a-1}^{W}\right)^{W+}$ are weighted pseudoinverses and $\mathbf{J}_{a}^{W *}$ is the weighted damped pseudoinverse with the weighting matrix $\mathbf{W}$. We recall that for a matrix $\mathbf{A} \in \mathbb{R}^{a \times b}$ and a symmetric and positive definite matrix $\mathbf{W} \in \mathbb{R}^{b \times b}$, the weighted pseudoinverse and the weighted damped pseudoinverse with a damping constant $\lambda \in(0, \infty)$ are defined as $\mathbf{A}^{W+}=$ $\mathbf{W}^{-1} \mathbf{A}^{T}\left(\mathbf{A} \mathbf{W}^{-1} \mathbf{A}^{T}\right)^{+}$and $\mathbf{A}^{W *}=\mathbf{W}^{-1} \mathbf{A}^{T}\left(\mathbf{A} \mathbf{W}^{-1} \mathbf{A}^{T}+\right.$ $\left.\lambda^{2} \mathbf{I}_{a}\right)^{-1}$, respectively. The idea of the weighted Chiaverini's solution is to precondition the linear inverse problem $\mathbf{J}(\mathbf{q}) \dot{\mathbf{q}}=$ $\mathbf{r}(t, \mathbf{q})$ by the right preconditioner $\mathbf{W}^{\frac{1}{2}}(\mathbf{q})$ for each $(t, \mathbf{q})$ such that the rows of $\left(\mathbf{J W}^{-\frac{1}{2}}\right)(\mathbf{q})$ are almost mutually orthogonal. So, if $\lambda_{1}=\cdots=\lambda_{l}=0$ and $0<\delta \ll \sigma_{\min }(\mathbf{J}(\mathbf{q}))$, then $\mathbf{e}^{\mathrm{res}}(t, \mathbf{q}) \approx 0$. Here, $\sigma_{\min }(\cdot)$ is the minimum singular value of a matrix.

Once we find a PIK solution $\mathbf{u}: \mathbb{R} \times \mathbb{R}^{n} \rightarrow \mathbb{R}^{n}$, we generate the joint trajectory $\mathbf{q}:\left[t_{0}, \infty\right) \rightarrow \mathbb{R}^{n}$ by solving the differential equation

$$
\dot{\mathbf{q}}=\mathbf{u}(t, \mathbf{q})
$$

for each $t \in\left(t_{0}, \infty\right)$ with the initial value $\mathbf{q}\left(t_{0}\right)=\mathbf{q}_{0}$. The first thing we must check is the smoothness of $\mathbf{u}$ because many important properties of the differential equation depend on it. For example, the solution of this initial value problem exists if $\mathbf{u}$ is continuous and linearly bounded $(\exists \gamma, c \in[0, \infty)$ : $\|\mathbf{u}(t, \mathbf{q})\| \leq \gamma\|\mathbf{q}\|+c, \forall(t, \mathbf{q}))$ and the solution is unique if 
$\mathbf{u}$ is locally Lipschitz and linearly bounded [35, pp.178]. So, a canonical question is: Can we always find a continuous or locally Lipschitz PIK solution? To answer this question, we first need to define the set of all PIK solutions.

For $\mathbf{A} \in \mathbb{R}^{m \times n}$, we define $\mathcal{R}(\mathbf{A})=\left\{\mathbf{A} \mathbf{b} \mid \mathbf{b} \in \mathbb{R}^{n}\right\}$ and $\mathcal{N}(\mathbf{A})=\left\{\mathbf{b} \in \mathbb{R}^{n} \mid \mathbf{A b}=\mathbf{0}\right\}$. We recall that $\mathcal{N}(\mathbf{A})^{\perp}=$ $\left\{\mathbf{c} \in \mathbb{R}^{n} \mid \mathbf{c}^{T} \mathbf{b}=0, \mathbf{b} \in \mathcal{N}(\mathbf{A})\right\}=\mathcal{R}\left(\mathbf{A}^{T}\right)$. For $a, b \in \mathbb{N}$, we use the shorthand notation $\overline{a, b}=\mathbb{N} \cap[a, b]$. We will need the following lemma later.

Lemma 1: Let $\mathbf{J} \in \mathbb{R}^{m \times n}$ with $m \leq n$. There exist a lower triangular matrix $\mathbf{C}_{e}=\left[c_{i j}\right] \in \mathbb{R}^{m \times n}$ and an orthogonal matrix $\hat{\mathbf{J}}_{e} \in \mathbb{R}^{n \times n}$ such that $\mathbf{J}=\mathbf{C}_{e} \hat{\mathbf{J}}_{e} ; c_{a a} \geq 0$ for $a \in \overline{1, m}$; and $c_{a b}=0$ for $a \in \overline{1, m}$ if $c_{b b}=0$.

Proof: See Appendix.

If $\mathbf{J} \in \mathbb{R}^{m \times n}$ has full rank, then the algorithm in the proof of Lemma 1 is same to the modified Gram-Schimidt orthogonalization that is numerically stable compared to the classical one [36]. If $\mathbf{J}$ is rank deficient, then the $\mathrm{QR}$ decomposition of $\mathbf{J}^{T}$ is not unique. Lemma 1 imposes an additional property $c_{a b}=0$ for $a \in \overline{1, m}$ if $c_{b b}=0$ that is important in this study. We remark that the QR decomposition of $\mathbf{J}^{T}$ by Householder reflectors does not give that property.

\section{GENERALIZATION}

Let $\mathbf{x}=(t, \mathbf{q})$ be a variable on $X=\mathbb{R} \times \mathbb{R}^{n}$. Generally, the $a$-th velocity mapping function is defined as $\mathbf{F}_{a}=$ $\left[\begin{array}{ll}\mathbf{f}_{t a} & \mathbf{F}_{q a}\end{array}\right]: X \rightarrow \mathbb{R}^{m_{a} \times(n+1)}$ with $\mathbf{f}_{t a}: X \rightarrow \mathbb{R}^{m_{a}}$ and $\mathbf{F}_{q a}: X \rightarrow \mathbb{R}^{m_{a} \times n}$ that maps the joint velocity $\dot{\mathbf{q}} \in \mathbb{R}^{n}$ into the $a$-th task velocity $\mathbf{f}_{t a}(\mathbf{x})+\mathbf{F}_{q a}(\mathbf{x}) \dot{\mathbf{q}} \in \mathbb{R}^{m_{a}}$ for each $\mathbf{x} \in X$. The $a$-th reference $\mathbf{r}_{a}: X \rightarrow \mathbb{R}^{m_{a}}$ is freely designed according to a scenario. Then, the goal of the $a$-th task at $\mathbf{x}$ is to find the best joint velocity $\dot{\mathbf{q}}$ that satisfies

$$
\mathbf{F}_{q a}(\mathbf{x}) \dot{\mathbf{q}}=\mathbf{r}_{a}(\mathbf{x})-\mathbf{f}_{t a}(\mathbf{x})=\mathbf{r}_{a}^{\prime}(\mathbf{x})
$$

at least approximately. Note that we need to solve $l$ such linear inverse problems simultaneously. Sometimes, the preconditioning of the matrix-valued functions $\mathbf{F}_{q 1}, \ldots, \mathbf{F}_{q l}$ gives better solvability. Let $\mathbf{R}: X \rightarrow \mathbb{R}^{n \times n}$ be invertible everywhere and define $\mathbf{J}_{a}: X \rightarrow \mathbb{R}^{m_{a} \times n}$ as $\mathbf{J}_{a}(\mathbf{x})=\mathbf{F}_{a}(\mathbf{x}) \mathbf{R}^{-1}(\mathbf{x})$. Then, the $a$-th linear inverse problem can be written as

$$
\mathbf{J}_{a}(\mathbf{x}) \mathbf{R}(\mathbf{x}) \dot{\mathbf{q}}=\mathbf{r}_{a}^{\prime}(\mathbf{x}) .
$$

The (right) preconditioner function $\mathbf{R}$ can be designed freely considering $\mathbf{F}_{q 1}, \ldots, \mathbf{F}_{q l}$. A specific choice of $\mathbf{R}$ and its effect on the PIK problem is discussed in [37]. One may let $\mathbf{R}=\mathbf{I}_{n}$ to ignore this part.

We define $\mathbf{f}_{t}=\left(\mathbf{f}_{t 1}, \ldots, \mathbf{f}_{t l}\right), \mathbf{F}_{q}=\left[\begin{array}{lll}\mathbf{F}_{q 1}^{T} & \cdots & \mathbf{F}_{q l}^{T}\end{array}\right]^{T}$, $\mathbf{F}=\left[\begin{array}{ll}\mathbf{f}_{t} & \mathbf{F}_{q}\end{array}\right], \mathbf{J}=\mathbf{F}_{q} \mathbf{R}^{-1}, \mathbf{r}=\left(\mathbf{r}_{1}, \ldots, \mathbf{r}_{l}\right)$, and $\mathbf{r}^{\prime}=\mathbf{r}-\mathbf{f}_{t}$. The PIK problem contains the orthogonalization process as a subproblem to find the projections into the null spaces of higher priority tasks. We orthogonalize rows of $\mathbf{J}$ by performing the full QR decomposition of $\mathbf{J}^{T}(\mathbf{x})$ at each $\mathbf{x} \in X$ as in Lemma 1

$$
\underbrace{\left[\begin{array}{c}
\mathbf{J}_{1} \\
\vdots \\
\mathbf{J}_{l}
\end{array}\right]}_{\mathbf{J}(\mathbf{x}) \in \mathbb{R}^{m \times n}}=\underbrace{\left[\begin{array}{cccc}
\mathbf{C}_{11} & \cdots & \mathbf{0} & \mathbf{0} \\
\vdots & \ddots & \vdots & \vdots \\
\mathbf{C}_{l 1} & \cdots & \mathbf{C}_{l l} & \mathbf{0}
\end{array}\right]}_{\mathbf{C}_{e}(\mathbf{x})=\left[\mathbf{C}_{i j}(\mathbf{x})\right] \in \mathbb{R}^{m \times n}} \underbrace{\left[\begin{array}{c}
\hat{\mathbf{J}}_{1} \\
\vdots \\
\hat{\mathbf{J}}_{l+1}
\end{array}\right]}_{\hat{\mathbf{J}}_{e}(\mathbf{x}) \in \mathbb{R}^{n \times n}} .
$$

Define orthogonal-projector-valued functions $\mathbf{P}_{a}: X \rightarrow$ $\mathbb{R}^{n \times n}$ for $a \in \overline{1, l}$ as

$$
\mathbf{P}_{a}(\mathbf{x})=\left(\left(\mathbf{C}_{a a} \hat{\mathbf{J}}_{a}\right)^{+}\left(\mathbf{C}_{a a} \hat{\mathbf{J}}_{a}\right)\right)(\mathbf{x})=\left(\hat{\mathbf{J}}_{a}^{T} \mathbf{C}_{a a}^{+} \mathbf{C}_{a a} \hat{\mathbf{J}}_{a}\right)(\mathbf{x}) .
$$

Then, $\mathbf{C}_{a b} \hat{\mathbf{J}}_{b}=\mathbf{J}_{a} \mathbf{P}_{b}$ by Lemma 1 and the $a$-th residual can be written as

$$
\mathbf{e}_{a}^{\mathrm{res}}=\mathbf{r}_{a}^{\prime}-\mathbf{J}_{a} \mathbf{R} \dot{\mathbf{q}}=\mathbf{r}_{a}^{\prime}-\mathbf{J}_{a} \sum_{b=1}^{a} \mathbf{P}_{b} \mathbf{R} \dot{\mathbf{q}} .
$$

Usually, multiple scenarios are applied for a mechanism in an environment, so we need to consider various references given $\mathbf{F}$ and $\mathbf{R}$. We expect that the inverse kinematics solution differs among various references. It leads us to think of the inverse kinematics solution as a function of the reference. Let $\left(\mathbb{R}^{m}\right)^{X}$ be the set of all functions from $X$ to $\mathbb{R}^{m}$. Then, we may define the inverse kinematics solution with multiple tasks considering various references as the best mapping $\mathbf{u}: X \times\left(\mathbb{R}^{m}\right)^{X} \rightarrow \mathbb{R}^{n}$ such that $\mathbf{v}=\mathbf{R u}$ satisfies $\mathbf{J}(\mathbf{x}) \mathbf{v}(\mathbf{x}, \mathbf{r})=\mathbf{r}^{\prime}(\mathbf{x})$ for each $(\mathbf{x}, \mathbf{r})$ at least approximately or equivalently minimizes the residual $\mathbf{e}^{\text {res }}(\mathbf{x}, \mathbf{y}, \mathbf{r})=\mathbf{r}^{\prime}(\mathbf{x})-$ $\mathbf{J}(\mathbf{x}) \mathbf{y}$ with respect to $\mathbf{y}$ for each $(\mathbf{x}, \mathbf{r})$ in some sense. Note that we extended the domain of the residual from $X \times \mathbb{R}^{n}$ to $X \times \mathbb{R}^{n} \times\left(\mathbb{R}^{m}\right)^{X}$ in order to consider various references. Here, 'at least approximately' and 'in some sense' can be understood very differently. We may specify the details on those expressions by constructing an optimization problem. Since we have multiple tasks, we need to introduce a vector-valued objective function $\boldsymbol{\pi}=\left(\pi_{1}, \ldots, \pi_{l}\right): X \times \mathbb{R}^{n} \times\left(\mathbb{R}^{m}\right)^{X} \rightarrow[0, \infty)^{l}$ that describes how to minimize $\left(\mathbf{e}_{1}^{\text {res }}, \ldots, \mathbf{e}_{l}^{\text {res }}\right)$ such that for each $\mathbf{r} \in\left(\mathbb{R}^{m}\right)^{X}$ the goal of the $a$-th task at $\mathbf{x}$ can be achieved by minimizing $\pi_{a}(\mathbf{x}, \mathbf{y}, \mathbf{r})$ with respect to $\mathbf{y}$. We remark that $\boldsymbol{\pi}$ is not a specific function but a variable of the function space that is defined as the set of all functions from $X \times \mathbb{R}^{n} \times\left(\mathbb{R}^{m}\right)^{X}$ to $[0, \infty)^{l}$. Not every objective function is appropriate for the PIK problem. For example, if $\pi_{a}(\mathbf{x}, \mathbf{y}, \mathbf{r})=\|\mathbf{y}\|$ for all $a \in \overline{1, l}$, then we have a trivial solution $\mathbf{u}=\mathbf{R}^{-1} \mathbf{v}=\mathbf{0}$. Therefore, we need to find properties for an objective function to be proper for the PIK problem.

(O1) Dependence: Since $\pi_{a}$ describes how to minimize $\mathbf{e}_{a}^{\mathrm{res}}(\mathbf{x}, \mathbf{y}, \mathbf{r})=\mathbf{r}_{a}^{\prime}(\mathbf{x})-\mathbf{J}_{a}(\mathbf{x}) \sum_{b=1}^{a} \mathbf{P}_{b}(\mathbf{x}) \mathbf{y}$ that depends on $\left(\mathbf{x}, \mathbf{y}, \mathbf{r}_{a}(\mathbf{x})\right)$, we may write $\pi_{a}(\mathbf{x}, \mathbf{y}, \mathbf{r})=\pi_{a}\left(\mathbf{x}, \mathbf{y}, \mathbf{r}_{a}(\mathbf{x})\right)$ for all $(\mathbf{x}, \mathbf{y}, \mathbf{r}) \in X \times \mathbb{R}^{n} \times\left(\mathbb{R}^{m}\right)^{X}$. Consider the minimization of $\pi_{a}\left(\mathbf{x}, \mathbf{y}, \mathbf{r}_{a}(\mathbf{x})\right)$ with respect to $\mathbf{y}$ and write $\mathbf{y}=\sum_{b=1}^{a} \mathbf{P}_{b}(\mathbf{x}) \mathbf{y}+\left(\mathbf{I}_{n}-\sum_{b=1}^{a} \mathbf{P}_{b}(\mathbf{x})\right) \mathbf{y}$. Only $\sum_{b=1}^{a} \mathbf{P}_{b}(\mathbf{x}) \mathbf{y}$ influences on $\mathbf{e}_{a}^{\text {res }}$ and there is a possibility that $\left(\mathbf{I}_{n}-\sum_{b=1}^{a} \mathbf{P}_{b}(\mathbf{x})\right) \mathbf{y}$ influences on $\mathbf{e}_{i}^{\text {res }}\left(\mathbf{x}, \mathbf{y}, \mathbf{r}_{i}(\mathbf{x})\right)$ for some $i>a$. Since the optimal value of $\mathbf{y}$ should be determined for all tasks, it is appropriate that $\pi_{a}\left(\mathbf{x}, \mathbf{y}, \mathbf{r}_{a}(\mathbf{x})\right)=$ $\pi_{a}\left(\mathbf{x}, \sum_{b=1}^{a} \mathbf{P}_{b}(\mathbf{x}) \mathbf{y}, \mathbf{r}_{a}(\mathbf{x})\right)$ for all $(\mathbf{x}, \mathbf{y}, \mathbf{r}) \in X \times \mathbb{R}^{n} \times$ $\left(\mathbb{R}^{m}\right)^{X}$. To better understand this property, let $\pi_{1}(\mathbf{x}, \mathbf{y}, \mathbf{r})=$ $\|\mathbf{y}\|$. Since the first task has the highest priority, we have a trivial solution $\mathbf{v}=\mathbf{0}$ regardless of the choice of $\pi_{2}, \ldots, \pi_{l}$ which is not desirable. On the other hand, if we let $\pi_{1}(\mathbf{x}, \mathbf{y}, \mathbf{r})=$ $\left\|\mathbf{P}_{1}(\mathbf{x}) \mathbf{y}\right\|$ that satisfies the property we demand, then we have $\mathbf{P}_{1} \mathbf{v}=\mathbf{0}$ and there is a possibility to choose $\mathbf{v}$ in the null space of $\mathbf{J}_{1}$ according to $\pi_{2}, \ldots, \pi_{l}$.

(O2) Uniqueness: If $\pi_{1}$ has the dependence property, then to minimize $\pi_{1}\left(\mathbf{x}, \mathbf{y}, \mathbf{r}_{1}(\mathbf{x})\right)$ subject to $\mathbf{y} \in \mathbb{R}^{n}$ is equivalent 
to minimize $\pi_{1}\left(\mathbf{x}, \mathbf{y}, \mathbf{r}_{1}(\mathbf{x})\right)$ subject to $\mathbf{y} \in \mathcal{R}\left(\mathbf{P}_{1}(\mathbf{x})\right)$. It is preferable that the minimizer of $\pi_{1}\left(\mathbf{x}, \mathbf{y}, \mathbf{r}_{1}(\mathbf{x})\right)$ subject to $\mathbf{y} \in \mathcal{R}\left(\mathbf{P}_{1}(\mathbf{x})\right)$ is unique not only to remove ambiguity in the selection of a minimizer but also to use the maximum available DOF for the first task. Let $\mathbf{y}_{1}^{*}$ be the unique minimizer of $\pi_{1}\left(\mathbf{x}, \mathbf{y}, \mathbf{r}_{1}(\mathbf{x})\right)$ subject to $\mathbf{y} \in \mathcal{R}\left(\mathbf{P}_{1}(\mathbf{x})\right)$ and consider the minimization of $\pi_{2}\left(\mathbf{x}, \mathbf{y}, \mathbf{r}_{2}(\mathbf{x})\right)$ with respect to $\mathbf{y}$ under the priority relations between tasks. Since the second priority task should not influence the first priority task, $\pi_{2}\left(\mathbf{x}, \mathbf{y}, \mathbf{r}_{2}(\mathbf{x})\right)$ should be minimized subject to $\mathbf{y} \in \mathbf{y}_{1}^{*}+\mathcal{R}\left(\mathbf{P}_{2}(\mathbf{x})\right)=$ $\left\{\mathbf{y}_{1}^{*}+\mathbf{z} \mid \mathbf{z} \in \mathcal{R}\left(\mathbf{P}_{2}(\mathbf{x})\right)\right\}$; it means that the minimizer of $\pi_{2}$ does not change the minimum value of $\pi_{1}$. By the dependence property, it is equivalent to minimize $\pi_{2}\left(\mathbf{x}, \mathbf{y}_{1}^{*}+\mathbf{y}, \mathbf{r}_{2}(\mathbf{x})\right)$ subject to $\mathbf{y} \in \mathcal{R}\left(\mathbf{P}_{2}(\mathbf{x})\right)$ and it is also preferable that the minimizer is unique by the same reason. We can continue the same reasoning for $a \in \overline{3, l}$ and find the expression of the uniqueness property: there exists a unique minimizer $\mathbf{y}_{a}^{*}$ of $\pi_{a}\left(\mathbf{x}, \sum_{b=1}^{a-1} \mathbf{y}_{b}^{*}+\mathbf{y}, \mathbf{r}_{a}(\mathbf{x})\right)$ subject to $\mathbf{y} \in \mathcal{R}\left(\mathbf{P}_{a}(\mathbf{x})\right)$.

(O3) Representation: Consider the mapping $\mathbf{r}_{a}(\mathbf{x}) \mapsto \mathbf{y}_{b}^{*}$ of $\mathbb{R}^{m_{a}}$ into $\mathcal{R}\left(\mathbf{P}_{b}(\mathbf{x})\right)$; it means the function $\mathbf{f}: \mathbb{R}^{m_{a}} \rightarrow$ $\mathcal{R}\left(\mathbf{P}_{b}(\mathbf{x})\right)$ defined as $\mathbf{f}\left(\mathbf{r}_{a}(\mathbf{x})\right)=\mathbf{y}_{b}^{*}$. Here, $\mathbf{r}_{a}(\mathbf{x})$ is considered as a variable on $\mathbb{R}^{m_{a}}$. If $b \in \overline{1, a-1}$, then $\mathbf{r}_{a}(\mathbf{x}) \mapsto \mathbf{y}_{b}^{*}$ is constant by the dependence and uniqueness properties because the minimizer $\mathbf{y}_{b}^{*}$ of $\pi_{b}$ is not influenced by the change of the $a$-th reference $\mathbf{r}_{a}$. Then, the minimization of $\pi_{a}\left(\mathbf{x}, \sum_{b=1}^{a-1} \mathbf{y}_{b}^{*}+\mathbf{y}, \mathbf{r}_{a}(\mathbf{x})\right)$ subject to $\mathbf{y} \in \mathcal{R}\left(\mathbf{P}_{a}(\mathbf{x})\right)$ can be considered as a way to solve the inverse problem $\mathbf{g}(\mathbf{y})=\left(\mathbf{C}_{a a} \hat{\mathbf{J}}_{a}\right)(\mathbf{x}) \mathbf{y}+\mathbf{J}_{a}(\mathbf{x}) \sum_{b=1}^{a-1} \mathbf{y}_{b}^{*}+\mathbf{f}_{t a}(\mathbf{x})=\mathbf{r}_{a}(\mathbf{x})$. Since $\mathcal{R}\left(\mathbf{P}_{a}(\mathbf{x})\right)=\mathcal{R}\left(\left(\mathbf{C}_{a a} \hat{\mathbf{J}}_{a}\right)^{T}(\mathbf{x})\right)=\mathcal{N}\left(\left(\mathbf{C}_{a a} \hat{\mathbf{J}}_{a}\right)(\mathbf{x})\right)^{\perp}$ (see the comment above Lemma 1), the linear transformation $\left(\mathbf{C}_{a a} \hat{\mathbf{J}}_{a}\right)(\mathbf{x})$ of $\mathcal{R}\left(\mathbf{P}_{a}(\mathbf{x})\right)$ into $\mathcal{R}\left(\left(\mathbf{C}_{a a} \hat{\mathbf{J}}_{a}\right)(\mathbf{x})\right)$ is one-to-one and onto by the Rank Theorem. We recall that a mapping $f: A \rightarrow B$ is said to be onto if $B=f(A)=\{f(a) \mid$ $a \in A\}$ and one-to-one if $f\left(a_{1}\right) \neq f\left(a_{2}\right)$ for every distinctive $a_{1}, a_{2} \in A$. If $f$ is one-to-one and onto, then there exists an inverse mapping $f^{-1}: B \rightarrow A$ satisfying $f\left(f^{-1}(b)\right)=b$ for all $b \in B$. So, we can find a one-to-one inverse mapping $\mathbf{g}^{-1}$ of $\mathcal{R}\left(\left(\mathbf{C}_{a a} \hat{\mathbf{J}}\right)(\mathbf{x})\right)$ onto $\mathcal{R}\left(\mathbf{P}_{a}(\mathbf{x})\right)$. This property is important because we expect that the joint velocities of two distinctive references that are realizable by the available DOF are different. So, it is desirable that the mapping $\mathbf{r}_{a}(\mathbf{x}) \mapsto \mathbf{y}_{a}^{*}$ of $\mathcal{R}\left(\left(\mathbf{C}_{a a} \hat{\mathbf{J}}_{a}\right)(\mathbf{x})\right)$ into $\mathcal{R}\left(\mathbf{P}_{a}(\mathbf{x})\right)$ is one-to-one and onto.

We say that a vector-valued objective function $\pi=$ $\left(\pi_{1}, \ldots, \pi_{l}\right): X \times \mathbb{R}^{n} \times\left(\mathbb{R}^{m}\right)^{X} \rightarrow[0, \infty)^{l}$ is proper for the PIK problem if $\pi$ has the following three properties:

(O1) $\forall(a, \mathbf{x}, \mathbf{y}, \mathbf{r}) \in \overline{1, l} \times X \times \mathbb{R}^{n} \times\left(\mathbb{R}^{m}\right)^{X}, \pi_{a}(\mathbf{x}, \mathbf{y}, \mathbf{r})=$ $\pi_{a}\left(\mathbf{x}, \sum_{b=1}^{a} \mathbf{P}_{b}(\mathbf{x}) \mathbf{y}, \mathbf{r}_{a}(\mathbf{x})\right)$

(O2) $\forall(a, \mathbf{x}, \mathbf{r}) \in \overline{1, l} \times X \times\left(\mathbb{R}^{m}\right)^{X}$, there exists a unique minimizer $\mathbf{y}_{a}^{*}$ of $\pi_{a}\left(\mathbf{x}, \sum_{b=1}^{a-1} \mathbf{y}_{b}^{*}+\mathbf{y}, \mathbf{r}_{a}(\mathbf{x})\right)$ subject to $\mathbf{y} \in \mathcal{R}\left(\mathbf{P}_{a}(\mathbf{x})\right)$;

(O3) $\forall(a, \mathbf{x}) \in \overline{1, l} \times X$, the mapping $\mathbf{r}_{a}(\mathbf{x}) \mapsto \mathbf{y}_{a}^{*}$ of $\mathcal{R}\left(\left(\mathbf{C}_{a a} \hat{\mathbf{J}}_{a}\right)(\mathbf{x})\right)$ into $\mathcal{R}\left(\mathbf{P}_{a}(\mathbf{x})\right)$ is one-to-one and onto.

The PIK problem is the process to find the PIK solution. We say that a map $\mathbf{u}: X \times\left(\mathbb{R}^{m}\right)^{X} \rightarrow \mathbb{R}^{n}$ is a PIK solution if there exists a proper objective function $\boldsymbol{\pi}=\left(\pi_{1}, \ldots, \pi_{l}\right)$ such that $\mathbf{R}(\mathbf{x}) \mathbf{u}(\mathbf{x}, \mathbf{r})$ minimizes $\pi_{a}(\mathbf{x}, \mathbf{y}, \mathbf{r})$ with respect to $\mathbf{y}$ for each $(a, \mathbf{x}, \mathbf{r}) \in \overline{1, l} \times X \times\left(\mathbb{R}^{m}\right)^{X}$ under the priority relations. The $\pi$-PIK solution is the PIK solution determined by the proper objective function $\pi$. We say that a PIK solution is smooth if $\mathbf{u}(\cdot, \mathbf{r})$ is smooth on $X$ for every smooth $\mathbf{r} \in\left(\mathbb{R}^{m}\right)^{X}$ and is nonsmooth if $\mathbf{u}(\cdot, \mathbf{r})$ is not smooth on $X$ for some smooth $\mathbf{r} \in\left(\mathbb{R}^{m}\right)^{X}$. Here, smoothness can be understood differently depending on the order of smoothness required; for example, it could be continuity, local Lipschitz continuity, continuous differentiability, etc.

The PIK problem can be written as the multi-objective optimization with the lexicographical ordering. Consider multiple objective functions $\phi_{a}: \mathbb{R}^{n} \rightarrow[0, \infty)$ for $a \in \overline{1, l}$ and a constraint set $\Omega \subset \mathbb{R}^{n}$. The problem

$$
\operatorname{lex}_{\mathbf{y} \in \Omega} \min \left(\phi_{1}(\mathbf{y}), \ldots, \phi_{l}(\mathbf{y})\right)
$$

is to find an optimal solution $\mathbf{y}^{*} \in \Omega$ satisfying

$$
\begin{aligned}
\phi_{a}\left(\mathbf{y}^{*}\right)=\min & \left\{\phi_{a}(\mathbf{y}) \mid \mathbf{y} \in \Omega\right. \text { and } \\
& \left.\phi_{b}(\mathbf{y})=\phi_{b}\left(\mathbf{y}^{*}\right) \text { for } b \in \overline{1, a-1}\right\}
\end{aligned}
$$

for all $a \in \overline{1, l}$. Let $\boldsymbol{\pi}: X \times \mathbb{R}^{n} \times\left(\mathbb{R}^{m}\right)^{X} \rightarrow[0, \infty)^{l}$ be a proper objective function. Fix $(\mathbf{x}, \mathbf{r}) \in X \times\left(\mathbb{R}^{m}\right)^{X}$ and define $\phi_{a}(\mathbf{y})=\pi_{a}(\mathbf{x}, \mathbf{y}, \mathbf{r})$ for $a \in \overline{1, l}$. By (O2), there exist $\mathbf{y}_{1}^{*}, \ldots, \mathbf{y}_{l}^{*} \in \mathbb{R}^{n}$ satisfying

$$
\begin{aligned}
\left\{\mathbf{y}_{1}^{*}\right\} & =\underset{\mathbf{y} \in \mathcal{R}\left(\mathbf{P}_{1}(\mathbf{x})\right)}{\arg \min } \phi_{1}(\mathbf{y}) \\
\left\{\mathbf{y}_{2}^{*}\right\} & =\underset{\mathbf{y} \in \mathcal{R}\left(\mathbf{P}_{2}(\mathbf{x})\right)}{\arg \min } \phi_{2}\left(\mathbf{y}_{1}^{*}+\mathbf{y}\right) \\
& \vdots \\
\left\{\mathbf{y}_{l}^{*}\right\} & =\underset{\mathbf{y} \in \mathcal{R}\left(\mathbf{P}_{l}(\mathbf{x})\right)}{\arg \min } \phi_{l}\left(\mathbf{y}_{1}^{*}+\cdots+\mathbf{y}_{l-1}^{*}+\mathbf{y}\right) .
\end{aligned}
$$

Let $\mathbf{y}^{*}=\mathbf{y}_{1}^{*}+\cdots+\mathbf{y}_{l}^{*}$. By $(\mathrm{O} 1), \phi_{a}\left(\mathbf{y}^{*}\right)=\phi_{a}\left(\mathbf{y}_{1}^{*}+\cdots+\mathbf{y}_{a}^{*}\right)$ for $a \in \overline{1, l}$ and

$$
\begin{aligned}
& \phi_{1}\left(\mathbf{y}^{*}\right)=\min \left\{\phi_{1}(\mathbf{y}) \mid \mathbf{y} \in \mathbb{R}^{n}\right\} \\
& \phi_{2}\left(\mathbf{y}^{*}\right)=\min \left\{\phi_{2}(\mathbf{y}) \mid \mathbf{y} \in \mathbf{y}_{1}^{*}+\mathcal{R}\left(\mathbf{I}_{n}-\mathbf{P}_{1}(\mathbf{x})\right)\right\} \\
&=\min \left\{\phi_{2}(\mathbf{y}) \mid \mathbf{y} \in \mathbb{R}^{n} \text { and } \phi_{1}(\mathbf{y})=\phi_{1}\left(\mathbf{y}_{1}^{*}\right)\right\} \\
& \vdots \\
& \phi_{l}\left(\mathbf{y}^{*}\right)=\min \left\{\phi_{l}(\mathbf{y}) \mid \mathbf{y} \in \sum_{b=1}^{l-1} \mathbf{y}_{b}^{*}+\mathcal{R}\left(\mathbf{I}_{n}-\sum_{b=1}^{l-1} \mathbf{P}_{b}(\mathbf{x})\right)\right\} \\
&= \min \left\{\phi_{l}(\mathbf{y}) \mid \mathbf{y} \in \mathbb{R}^{n}\right. \text { and } \\
&\left.\quad \phi_{b}(\mathbf{y})=\phi_{b}\left(\mathbf{y}^{*}\right) \text { for } b \in \overline{1, l-1}\right\} \\
& \frac{\left\|\mathbf{y}^{*}\right\|^{2}}{2}=\min \left\{\|\mathbf{y}\|^{2} / 2 \mid \mathbf{y} \in \mathbb{R}^{n}\right. \text { and } \\
&\left.\quad \phi_{b}(\mathbf{y})=\phi_{b}\left(\mathbf{y}^{*}\right) \text { for } b \in \overline{1, l}\right\}
\end{aligned}
$$

$\mathbf{y}^{*}$ minimizes $\pi_{a}(\mathbf{x}, \mathbf{y}, \mathbf{r})$ with respect to $\mathbf{y}$ for $a \in \overline{1, l}$ under the priority relations and is a unique solution of

$$
\underset{\mathbf{y} \in \mathbb{R}^{n}}{\operatorname{lex} \min }\left(\pi_{1}(\mathbf{x}, \mathbf{y}, \mathbf{r}), \ldots, \pi_{l}(\mathbf{x}, \mathbf{y}, \mathbf{r}),\|\mathbf{y}\|^{2} / 2\right) .
$$

Therefore, we can write the $\pi$-PIK solution as

$$
\begin{aligned}
& \mathbf{u}(\mathbf{x}, \mathbf{r})=\mathbf{R}^{-1}(\mathbf{x}) \mathbf{v}(\mathbf{x}, \mathbf{r}) \\
& \mathbf{v}(\mathbf{x}, \mathbf{r}) \in \underset{\mathbf{y} \in \mathbb{R}^{n}}{\arg \operatorname{lex} \min }\left(\pi_{1}(\mathbf{x}, \mathbf{y}, \mathbf{r}), \cdots, \pi_{l}(\mathbf{x}, \mathbf{y}, \mathbf{r}),\|\mathbf{y}\|^{2} / 2\right)
\end{aligned}
$$


We call a function $\mathbf{u}$ satisfying the above problem with an objective function $\pi$ a $\pi$-PIK solution candidate when the properness of $\pi$ is not evaluated.

Let $\Pi$ be the set of all functions from $X \times \mathbb{R}^{n} \times\left(\mathbb{R}^{m}\right)^{X}$ to $[0, \infty)^{l}, U$ be the set of all functions from $X \times\left(\mathbb{R}^{m}\right)^{X}$ to $\mathbb{R}^{n}$, and $\theta: \Pi \rightarrow U$ be the function defined as (7). Let $\Pi_{p}$ and $U_{p}$ be the set of all proper objective functions and the set of all PIK solutions, respectively. Then, we can compactly represent our generalization of the PIK problem as

$$
U_{p}=\theta\left(\Pi_{p}\right) .
$$

In other words, the set of all PIK solutions is generated from the set of all proper objective functions by the mapping defined as the optimal solution of the multi-objective optimization with the lexicographical ordering. At this point, we must clarify that (8) is not the only way to generalize the PIK problem. One can freely propose a different notion of generalization and study the relations with our generalization. Also, (8) does not provide a way to find all PIK solutions but a way to study theoretical properties of the PIK problem. One important property we found is nonsmoothness of PIK solutions defined by (8). Once a PIK solution $\mathbf{u}$ is determined from $U_{p}$, we generate a joint trajectory $\mathbf{q}:\left[t_{0}, \infty\right) \rightarrow \mathbb{R}^{n}$ with an initial value $\mathbf{q}\left(t_{0}\right)=\mathbf{q}_{0}$ and a reference $\mathbf{r}$ by solving the differential equation

$$
\dot{\mathbf{q}}(t)=\mathbf{u}(t, \mathbf{q}(t), \mathbf{r}), t \in\left(t_{0}, \infty\right) .
$$

As we discussed in Section II, smoothness of $\mathbf{u}$ given $\mathbf{r}$ is an important property we must check. Now, the question "Can we always find a smooth PIK solution given smooth Jacobians and references?" can be interpreted as "For every smooth $\mathbf{F}$ and $\mathbf{R}$, does there exist a proper objective function $\boldsymbol{\pi}$ such that the $\boldsymbol{\pi}$-PIK solution is smooth for all smooth $\mathbf{r}$ ?". Unfortunately, our analysis shows that if smooth $\mathbf{F}$ and $\mathbf{R}$ satisfies a certain condition, then for every $\boldsymbol{\pi} \in \Pi_{p}$ there exists smooth $\mathbf{r}$ such that $\mathbf{u}=\theta(\boldsymbol{\pi})$ given $\mathbf{r}$ is nonsmooth [38]. This analysis result sends an important message that prioritization can induce nonsmoothness, so that we need to put our efforts to construct an alternative existence theorem of the joint trajectory, other than classical existence theorems. In the rest of this letter, we furnish some examples of PIK solutions to confirm that our generalization is reasonable.

\section{EXAMPLES}

We start by showing that our generalization discards some trivial solutions. Assume that $\operatorname{rank}\left(\mathbf{J}_{1}\left(\mathbf{x}_{0}\right)\right)>0$ for $\mathbf{x}_{0} \in X$. Define $\pi_{1}(\mathbf{x}, \mathbf{y}, \mathbf{r})=\|\mathbf{g}(\mathbf{x})-\mathbf{y}\|$ for some $\mathbf{g}: X \rightarrow \mathbb{R}^{n}$. Then, for every $\pi_{2}, \ldots, \pi_{l}$ we have a unique $\pi$-PIK solution candidate $\mathbf{u}(\mathbf{x}, \mathbf{r})=\mathbf{R}^{-1}(\mathbf{x}) \mathbf{g}(\mathbf{x})$. Indeed, it is not a PIK solution because $\pi_{1}$ we chose does not have the properties (O1) and (O3) at $\mathbf{x}_{0}$. Intuitively speaking, a proper objective function should not constrain lower priority tasks unnecessarily and should give a different PIK solution if we change the reference that is realizable by the available DOFs. So, a constant function such as $\mathbf{u}=\mathbf{0}$ is not a PIK solution unless $\mathbf{J}=\mathbf{0}$ in our generalization.

Next, we show that our generalization includes two successful PIK solutions. Define vector-valued objective functions $\boldsymbol{\pi}_{\alpha}^{\times}=\left(\pi_{\alpha 1}^{\times}, \ldots, \pi_{\alpha l}^{\times}\right): X \times \mathbb{R}^{n} \times\left(\mathbb{R}^{m}\right)^{X} \rightarrow[0, \infty)^{l}$ for $\alpha \in \overline{1,2}$ and $\times \in\{+, *\}$ as:

$$
\begin{aligned}
& \pi_{1 a}^{+}(\mathbf{x}, \mathbf{y}, \mathbf{r})=\frac{1}{2}\left\|\mathbf{r}_{a}^{\prime}(\mathbf{x})-\mathbf{J}_{a}(\mathbf{x}) \mathbf{y}\right\|^{2} \\
& \pi_{1 a}^{*}(\mathbf{x}, \mathbf{y}, \mathbf{r})=\frac{1}{2}\left\|\mathbf{r}_{a}^{\prime}(\mathbf{x})-\mathbf{J}_{a}(\mathbf{x}) \mathbf{y}\right\|^{2}+\frac{1}{2} \lambda_{a}^{2}(\mathbf{x})\left\|\mathbf{P}_{a}(\mathbf{x}) \mathbf{y}\right\|^{2} \\
& \pi_{2 a}^{+}(\mathbf{x}, \mathbf{y}, \mathbf{r})=\frac{1}{2}\left\|\mathbf{J}_{a}^{+}(\mathbf{x}) \mathbf{r}_{a}^{\prime}(\mathbf{x})-\mathbf{P}_{a}(\mathbf{x}) \mathbf{y}\right\|^{2} \\
& \pi_{2 a}^{*}(\mathbf{x}, \mathbf{y}, \mathbf{r})=\frac{1}{2}\left\|\mathbf{J}_{a}^{*}(\mathbf{x}) \mathbf{r}_{a}^{\prime}(\mathbf{x})-\mathbf{P}_{a}(\mathbf{x}) \mathbf{y}\right\|^{2}
\end{aligned}
$$

where the damping function $\lambda_{a}: X \rightarrow(0, \infty)$ is arbitrary and $\mathbf{J}_{a}^{*}(\mathbf{x})$ is the damped pseudoinverse of $\mathbf{J}_{a}(\mathbf{x})$ with the damping constant $\lambda_{a}(\mathbf{x})$ at $\mathbf{x}$. Objective functions have the property (O1) because $\mathbf{J}_{a}=\mathbf{J}_{a} \sum_{b=1}^{a} \mathbf{P}_{b}$ by Lemma 1 . Since $\mathcal{R}\left(\mathbf{P}_{a}(\mathbf{x})\right)=\mathcal{R}\left(\left(\mathbf{C}_{a a} \hat{\mathbf{J}}_{a}\right)^{T}(\mathbf{x})\right)=\mathcal{N}\left(\left(\mathbf{C}_{a a} \hat{\mathbf{J}}_{a}\right)(\mathbf{x})\right)^{\perp}$ for all $(a, \mathbf{x}) \in \overline{1, l} \times X$, the minimization problem

$$
\begin{aligned}
& \min \frac{1}{2}\left\|\mathbf{r}_{1}^{\prime}(\mathbf{x})-\left(\mathbf{C}_{11} \hat{\mathbf{J}}_{1}\right)(\mathbf{x}) \mathbf{y}\right\|^{2} \\
& \text { s.t. } \mathbf{y} \in \mathcal{R}\left(\mathbf{P}_{1}(\mathbf{x})\right)
\end{aligned}
$$

has a unique solution $\mathbf{y}_{1}^{*}=\left(\hat{\mathbf{J}}_{1}^{T} \mathbf{C}_{11}^{+} \mathbf{r}_{1}^{\prime}\right)(\mathbf{x})$. It follows that the minimization problem

$$
\begin{aligned}
& \min \frac{1}{2}\left\|\left(\mathbf{r}_{a}^{\prime}-\mathbf{J}_{a} \sum_{b=1}^{a-1} \mathbf{y}_{b}^{*}\right)(\mathbf{x})-\left(\mathbf{C}_{a a} \hat{\mathbf{J}}_{a}\right)(\mathbf{x}) \mathbf{y}\right\|^{2} \\
& \text { s.t. } \mathbf{y} \in \mathcal{R}\left(\mathbf{P}_{a}(\mathbf{x})\right)
\end{aligned}
$$

has a unique solution $\mathbf{y}_{a}^{*}=\left(\hat{\mathbf{J}}_{a}^{T} \mathbf{C}_{a a}^{+}\left(\mathbf{r}_{a}^{\prime}-\mathbf{J}_{a} \sum_{b=1}^{a-1} \mathbf{y}_{b}^{*}\right)\right)(\mathbf{x})$ for $a \in \overline{1, l}$. It is obvious that the replacement of $\mathbf{r}_{a}(\mathbf{x})$ with $\tilde{\mathbf{r}}_{a}(\mathbf{x}) \in \mathbb{R}^{m_{a}}$ does not change $\mathbf{y}_{b}^{*}$ for $b \in \overline{1, a-1}$. Let $\mathbf{r}_{a}(\mathbf{x}), \tilde{\mathbf{r}}_{a}(\mathbf{x}) \in \mathcal{R}\left(\left(\mathbf{C}_{a a} \hat{\mathbf{J}}_{a}\right)(\mathbf{x})\right)=\mathcal{R}\left(\mathbf{C}_{a a}(\mathbf{x})\right), \mathbf{r}_{a}(\mathbf{x}) \neq$ $\tilde{\mathbf{r}}_{a}(\mathbf{x})$, and $\tilde{\mathbf{y}}_{a}^{*}=\left(\hat{\mathbf{J}}_{a}^{T} \mathbf{C}_{a a}^{+}\left(\tilde{\mathbf{r}}_{a}-\mathbf{F}_{t a}-\mathbf{J}_{a} \sum_{b=1}^{a-1} \mathbf{y}_{b}^{*}\right)\right)(\mathbf{x})$. $\mathbf{0} \neq\left(\mathbf{r}_{a}-\tilde{\mathbf{r}}_{a}\right)(\mathbf{x}) \in \mathcal{R}\left(\mathbf{C}_{a a}(\mathbf{x})\right)$ and $\mathcal{N}\left(\mathbf{C}_{a a}^{+}(\mathbf{x})\right)=$ $\mathcal{R}\left(\mathbf{C}_{a a}(\mathbf{x})\right)^{\perp}$ imply that $\left(\mathbf{C}_{a a}^{+}\left(\mathbf{r}_{a}-\tilde{\mathbf{r}}_{a}\right)\right)(\mathbf{x}) \neq \mathbf{0}$ and $\mathbf{y}_{a}^{*} \neq \tilde{\mathbf{y}}_{a}^{*}$. Since the mapping $\mathbf{r}_{a}(\mathbf{x}) \mapsto \mathbf{y}_{a}^{*}$ is affine and $\operatorname{dim}\left(\mathcal{R}\left(\left(\mathbf{C}_{a a} \hat{\mathbf{J}}_{a}\right)(\mathbf{x})\right)\right)=\operatorname{dim}\left(\mathcal{R}\left(\mathbf{P}_{a}(\mathbf{x})\right)\right)$, the mapping $\mathbf{r}_{a}(\mathbf{x}) \mapsto \mathbf{y}_{a}^{*}$ of $\mathcal{R}\left(\left(\mathbf{C}_{a a} \hat{\mathbf{J}}_{a}\right)(\mathbf{x})\right)$ into $\mathcal{R}\left(\mathbf{P}_{a}(\mathbf{x})\right)$ is one-toone and onto where $\operatorname{dim}(\cdot)$ is the dimension of a vector space. So, $\boldsymbol{\pi}_{1}^{+}$is proper. By letting $\mathbf{v}_{a}(\mathbf{x}, \mathbf{r})=\mathbf{y}_{a}^{*}$ for each $(a, \mathbf{x}, \mathbf{r}) \in \overline{1, l} \times X \times\left(\mathbb{R}^{m}\right)^{X}$, we can derive the recursive form $\boldsymbol{\pi}_{1}^{+}$-PIK solution as

$$
\begin{aligned}
\mathbf{u} & =\mathbf{R}^{-1} \mathbf{v}_{1: l} \\
\mathbf{v}_{1: a} & =\mathbf{v}_{1: a-1}+\hat{\mathbf{J}}_{a}^{T} \mathbf{C}_{a a}^{+}\left(\mathbf{r}_{a}^{\prime}-\mathbf{J}_{a} \mathbf{v}_{1: a-1}\right) \\
\mathbf{v}_{1: 0} & =\mathbf{0}
\end{aligned}
$$

where $\mathbf{v}_{1: a}=\mathbf{v}_{1}+\cdots+\mathbf{v}_{a}$ for $a \in \overline{1, l}$.

We also find the closed form $\boldsymbol{\pi}_{1}^{+}$-PIK solution. Define C $: X \rightarrow \mathbb{R}^{m \times m}$ as the left $(m \times m)$ block of $\mathbf{C}_{e}$ and $\hat{\mathbf{J}}: X \rightarrow \mathbb{R}^{m \times n}$ as the top $(m \times n)$ block of $\hat{\mathbf{J}}_{e}$ such that $\mathbf{J}=\mathbf{C}_{e} \hat{\mathbf{J}}_{e}=\mathbf{C} \hat{\mathbf{J}}$. Note that $\mathbf{J}^{T}=\hat{\mathbf{J}}^{T} \mathbf{C}^{T}$ is the reduced $\mathrm{QR}$ decomposition of $\mathbf{J}^{T}$ when $m<n$. Define $\mathbf{C}_{D}=\operatorname{diag}\left(\mathbf{C}_{11}, \ldots, \mathbf{C}_{l l}\right)$ and $\mathbf{C}_{L}=\mathbf{C}-\mathbf{C}_{D}$ where $\operatorname{diag}\left(\mathbf{C}_{11}, \ldots, \mathbf{C}_{l l}\right)$ is the block diagonal matrix whose diagonal blocks are $\mathbf{C}_{11}, \ldots, \mathbf{C}_{l l}$ starting from the top left 
corner. Obviously, $\mathbf{C}_{D}^{+}=\operatorname{diag}\left(\mathbf{C}_{11}^{+}, \ldots, \mathbf{C}_{l l}^{+}\right)$. We can rewrite $\mathbf{v}=\mathbf{v}_{1}+\cdots+\mathbf{v}_{l}$ as

$$
\mathbf{v}=\hat{\mathbf{J}}^{T}\left[\begin{array}{c}
\mathbf{C}_{11}^{+} \mathbf{r}_{1}^{\prime} \\
\mathbf{C}_{22}^{+}\left(\mathbf{r}_{2}^{\prime}-\mathbf{C}_{21} \hat{\mathbf{J}}_{1} \mathbf{v}_{1}\right) \\
\vdots \\
\mathbf{C}_{l l}^{+}\left(\mathbf{r}_{l}^{\prime}-\sum_{b=1}^{l-1} \mathbf{C}_{l b} \hat{\mathbf{J}}_{b} \mathbf{v}_{b}\right)
\end{array}\right]=\hat{\mathbf{J}}^{T} \mathbf{z}
$$

using $\mathbf{J}_{a} \sum_{b=1}^{a-1} \mathbf{v}_{b}=\sum_{b=1}^{a-1} \mathbf{C}_{a b} \hat{\mathbf{J}}_{b} \mathbf{v}_{b}$. By extracting $\mathbf{C}_{a a}^{+}$from $\mathbf{z}$, we find $\mathbf{z}=\mathbf{C}_{D}^{+}\left(\mathbf{r}^{\prime}-\mathbf{C}_{L} \mathbf{z}\right)$ and $\mathbf{z}=\left(\mathbf{I}_{m}+\mathbf{C}_{D}^{+} \mathbf{C}_{L}\right)^{-1} \mathbf{C}_{D}^{+} \mathbf{r}^{\prime}$. Since $\mathbf{C}_{D}^{+} \mathbf{C}_{L}$ and $\mathbf{C}_{L} \mathbf{C}_{D}^{+}$are strictly block lower triangular, we have

$$
\begin{aligned}
& \left(\mathbf{I}_{m}+\mathbf{C}_{D}^{+} \mathbf{C}_{L}\right)^{-1} \mathbf{C}_{D}^{+} \\
& =\left[\mathbf{I}_{m}-\mathbf{C}_{D}^{+} \mathbf{C}_{L}+\cdots+\left(-\mathbf{C}_{D}^{+} \mathbf{C}_{L}\right)^{l-1}\right] \mathbf{C}_{D}^{+} \\
& =\mathbf{C}_{D}^{+}\left[\mathbf{I}_{m}-\mathbf{C}_{L} \mathbf{C}_{D}^{+}+\cdots+\left(-\mathbf{C}_{L} \mathbf{C}_{D}^{+}\right)^{l-1}\right] \\
& =\mathbf{C}_{D}^{+}\left(\mathbf{I}_{m}+\mathbf{C}_{L} \mathbf{C}_{D}^{+}\right)^{-1} .
\end{aligned}
$$

We call $\mathbf{C}^{\oplus}=\left(\mathbf{I}_{m}+\mathbf{C}_{D}^{+} \mathbf{C}_{L}\right)^{-1} \mathbf{C}_{D}^{+}=\mathbf{C}_{D}^{+}\left(\mathbf{I}_{m}+\mathbf{C}_{L} \mathbf{C}_{D}^{+}\right)^{-1}$ as the prioritized pseudoinverse of $\mathbf{C}$. It gives the closed form $\boldsymbol{\pi}_{1}^{+}$-PIK solution as

$$
\mathbf{u}=\mathbf{R}^{-1} \hat{\mathbf{J}}^{T} \mathbf{C}^{\oplus} \mathbf{r}^{\prime}
$$

From $\mathcal{R}\left(\mathbf{P}_{a}(\mathbf{x})\right)=\mathcal{N}\left(\left(\mathbf{C}_{a a} \hat{\mathbf{J}}_{a}\right)(\mathbf{x})\right)^{\perp}$ and $\mathbf{C}_{a a} \hat{\mathbf{J}}_{a}=$ $\mathbf{C}_{a a} \hat{\mathbf{J}}_{a} \mathbf{P}_{a}$, we can find a unique solution of the minimization problem

$$
\begin{aligned}
& \min \frac{1}{2}\left\|\mathbf{r}_{1}^{\prime}(\mathbf{x})-\left(\mathbf{C}_{11} \hat{\mathbf{J}}_{1}\right)(\mathbf{x}) \mathbf{y}\right\|^{2}+\frac{1}{2} \lambda_{1}^{2}(\mathbf{x})\left\|\mathbf{P}_{1}(\mathbf{x}) \mathbf{y}\right\|^{2} \\
& \text { s.t. } \mathbf{y} \in \mathcal{R}\left(\mathbf{P}_{1}(\mathbf{x})\right)
\end{aligned}
$$

as $\mathbf{y}_{1}^{*}=\left(\hat{\mathbf{J}}_{1}^{T} \mathbf{C}_{11}^{*} \mathbf{r}_{1}^{\prime}\right)(\mathbf{x})$ where $\mathbf{C}_{a a}^{*}$ is the damped pseudoinverse of $\mathbf{C}_{a a}$ as before. It follows that the minimization problem

$$
\begin{aligned}
\min \frac{1}{2}\left\|\left(\mathbf{r}_{a}^{\prime}-\mathbf{J}_{a} \sum_{b=1}^{a-1} \mathbf{y}_{b}^{*}\right)(\mathbf{x})-\left(\mathbf{C}_{a a} \hat{\mathbf{J}}_{a}\right)(\mathbf{x}) \mathbf{y}\right\|^{2} \\
+\frac{1}{2} \lambda_{a}^{2}(\mathbf{x})\left\|\mathbf{P}_{a}(\mathbf{x}) \mathbf{y}\right\|^{2} \\
\text { s.t. } \mathbf{y} \in \mathcal{R}\left(\mathbf{P}_{a}(\mathbf{x})\right)
\end{aligned}
$$

has a unique solution $\mathbf{y}_{a}^{*}=\left(\hat{\mathbf{J}}_{a}^{T} \mathbf{C}_{a a}^{*}\left(\mathbf{r}_{a}^{\prime}-\mathbf{J}_{a} \sum_{b=1}^{a-1} \mathbf{y}_{b}^{*}\right)\right)(\mathbf{x})$ for $a \in \overline{1, l}$. Similarly as before, (O3) can be checked from $\mathcal{N}\left(\mathbf{C}_{a a}^{*}(\mathbf{x})\right)=\mathcal{R}\left(\mathbf{C}_{a a}(\mathbf{x})\right)^{\perp}$. Hence, $\boldsymbol{\pi}_{1}^{*}$ is proper. Define $\mathbf{C}_{D}^{\circledast}=\operatorname{diag}\left(\mathbf{C}_{11}^{*}, \ldots, \mathbf{C}_{l l}^{*}\right)$. We call $\mathbf{C}^{\circledast}=\mathbf{C}_{D}^{\circledast}\left(\mathbf{I}_{m}+\right.$ $\left.\mathbf{C}_{L} \mathbf{C}_{D}^{\circledast}\right)^{-1}=\left(\mathbf{I}_{m}+\mathbf{C}_{D}^{\circledast} \mathbf{C}_{L}\right)^{-1} \mathbf{C}_{D}^{\circledast}$ as the prioritized damped pseudoinverse of $\mathbf{C}$. It is straightforward to derive the $\pi_{1}^{*}$-PIK solution in the recursive form

$$
\begin{aligned}
\mathbf{u} & =\mathbf{R}^{-1} \mathbf{v}_{1: l} \\
\mathbf{v}_{1: a} & =\mathbf{v}_{1:(a-1)}+\hat{\mathbf{J}}_{a}^{T} \mathbf{C}_{a a}^{*}\left(\mathbf{r}_{a}^{\prime}-\mathbf{J}_{a} \mathbf{v}_{1:(a-1)}\right) \\
\mathbf{v}_{1: 0} & =\mathbf{0}
\end{aligned}
$$

and in the closed form

$$
\mathbf{u}=\mathbf{R}^{-1} \hat{\mathbf{J}}^{T} \mathbf{C}^{\circledast} \mathbf{r}^{\prime} .
$$

It is immediate to check that $\boldsymbol{\pi}_{2}^{+}$and $\boldsymbol{\pi}_{2}^{*}$ are proper and to derive the $\pi_{2}^{+}$-PIK solution as

$$
\mathbf{u}=\mathbf{R}^{-1} \sum_{a=1}^{l} \hat{\mathbf{J}}_{a}^{T} \mathbf{C}_{a a}^{T}\left(\mathbf{J}_{a} \mathbf{J}_{a}^{T}\right)^{+} \mathbf{r}_{a}^{\prime}=\mathbf{R}^{-1} \hat{\mathbf{J}}^{T} \mathbf{C}_{D}^{T} \mathbf{H}_{+} \mathbf{r}^{\prime}
$$

and the $\pi_{2}^{*}$-PIK solution as

$\mathbf{u}=\mathbf{R}^{-1} \sum_{a=1}^{l} \hat{\mathbf{J}}_{a}^{T} \mathbf{C}_{a a}^{T}\left(\mathbf{J}_{a} \mathbf{J}_{a}^{T}+\lambda_{a}^{2} \mathbf{I}_{m_{a}}\right)^{-1} \mathbf{r}_{a}^{\prime}=\mathbf{R}^{-1} \hat{\mathbf{J}}^{T} \mathbf{C}_{D}^{T} \mathbf{H}_{*} \mathbf{r}^{\prime}$

by using $\mathbf{J}_{a} \mathbf{P}_{a}=\mathbf{J}_{a} \hat{\mathbf{J}}_{a}^{T} \mathbf{C}_{a a}^{+} \mathbf{C}_{a a} \hat{\mathbf{J}}_{a}=\mathbf{C}_{a a} \hat{\mathbf{J}}_{a}$ where $\mathbf{H}_{+}=$ $\operatorname{diag}\left(\left(\mathbf{J}_{1} \mathbf{J}_{1}^{T}\right)^{+}, \ldots,\left(\mathbf{J}_{l} \mathbf{J}_{l}^{T}\right)^{+}\right)$and $\mathbf{H}_{*}=\operatorname{diag}\left(\left(\mathbf{J}_{1} \mathbf{J}_{1}^{T}+\right.\right.$ $\left.\left.\lambda_{1}^{2} \mathbf{I}_{m_{1}}\right)^{-1}, \ldots,\left(\mathbf{J}_{l} \mathbf{J}_{l}^{T}+\lambda_{l}^{2} \mathbf{I}_{m_{l}}\right)^{-1}\right)$.

Now, define $\mathbf{N}_{a}=\mathbf{I}_{n}-\sum_{b=1}^{a} \mathbf{P}_{b}$ for $a \in \overline{1, l}$ and $\mathbf{N}_{0}=\mathbf{I}_{n}$. $\mathbf{J}_{1} \mathbf{N}_{0}=\mathbf{C}_{11} \hat{\mathbf{J}}_{1}$ and $\mathbf{N}_{1}=\mathbf{N}_{0}-\left(\mathbf{J}_{1} \mathbf{N}_{0}\right)^{+}\left(\mathbf{J}_{1} \mathbf{N}_{0}\right)$. Also, $\mathbf{J}_{a} \mathbf{N}_{a-1}=\left(\mathbf{C}_{a 1} \hat{\mathbf{J}}_{1}+\cdots+\mathbf{C}_{a a} \hat{\mathbf{J}}_{a}\right)\left(\mathbf{I}_{n}-\sum_{b=1}^{a-1} \mathbf{P}_{a}\right)=\mathbf{C}_{a a} \hat{\mathbf{J}}_{a}$ and $\mathbf{N}_{a}=\mathbf{N}_{a-1}-\left(\mathbf{J}_{a} \mathbf{N}_{a-1}\right)^{+}\left(\mathbf{J}_{a} \mathbf{N}_{a-1}\right)$ for $a \in \overline{2, l}$ by Lemma 1 . Then, we can rewrite the $\boldsymbol{\pi}_{1}^{+}$-PIK solution as

$$
\begin{aligned}
\mathbf{u} & =\mathbf{R}^{-1} \mathbf{v}_{1: l} \\
\mathbf{v}_{1: a} & =\mathbf{v}_{1: a-1}+\left(\mathbf{J}_{a} \mathbf{N}_{a-1}\right)^{+}\left(\mathbf{r}_{a}^{\prime}-\mathbf{J}_{a} \mathbf{v}_{1: a-1}\right) \\
\mathbf{v}_{1: 0} & =\mathbf{0}
\end{aligned}
$$

and the $\boldsymbol{\pi}_{1}^{*}$-PIK solution by replacing $\mathbf{v}_{1: a}$ with

$$
\mathbf{v}_{1: a}=\mathbf{v}_{1: a-1}+\left(\mathbf{J}_{a} \mathbf{N}_{a-1}\right)^{*}\left(\mathbf{r}_{a}^{\prime}-\mathbf{J}_{a} \mathbf{v}_{1: a-1}\right)
$$

that are same to the Nakamura's solution (1) and (2) except for the preconditioning. Also, we can rewrite the $\pi_{2}^{+}$-PIK solution as

$$
\mathbf{u}=\mathbf{R}^{-1} \sum_{a=1}^{l} \mathbf{N}_{a-1} \mathbf{J}_{a}^{+} \mathbf{r}_{a}^{\prime}
$$

and the $\pi_{2}^{*}$-PIK solution as

$$
\mathbf{u}=\mathbf{R}^{-1} \sum_{a=1}^{l} \mathbf{N}_{a-1} \mathbf{J}_{a}^{*} \mathbf{r}_{a}^{\prime}
$$

that are same to the Chiaverini's solution (3) and (4) except for the preconditioning. If we let $\mathbf{R}=\left(\mathbf{F}_{q}^{T} \mathbf{F}_{q}+\delta^{2} \mathbf{I}_{n}\right)^{\frac{1}{2}}=\mathbf{W}^{\frac{1}{2}}$ with $\delta \in(0, \infty)$, then we can check that the $\boldsymbol{\pi}_{2}^{\times}$-PIK solution is same to the weighted Chiaverini's solution (5) and (6) from $\mathbf{N}_{a}^{W}=\mathbf{W}^{-\frac{1}{2}} \mathbf{N}_{a} \mathbf{W}^{\frac{1}{2}}$ and $\mathbf{N}_{a-1}^{W} \mathbf{F}_{q a}^{W \times}=\mathbf{R}^{-1} \mathbf{N}_{a-1} \mathbf{J}_{a}^{\times}$.

We can come up with other PIK solutions. Define objective functions $\boldsymbol{\pi}_{3}^{\times}, \boldsymbol{\pi}_{4}: X \times \mathbb{R}^{n} \times\left(\mathbb{R}^{m}\right)^{X} \rightarrow[0, \infty)^{l}$ as:

$$
\begin{aligned}
\pi_{3 a}^{+}(\mathbf{x}, \mathbf{y}, \mathbf{r}) & =\frac{1}{2}\left\|\mathbf{r}_{a}^{\prime}(\mathbf{x})-\left(\mathbf{C}_{a a} \hat{\mathbf{J}}_{a}\right)(\mathbf{x}) \mathbf{y}\right\|^{2} \\
\pi_{3 a}^{*}(\mathbf{x}, \mathbf{y}, \mathbf{r}) & =\pi_{3 a}^{+}(\mathbf{x}, \mathbf{y}, \mathbf{r})+\frac{1}{2} \lambda_{a}^{2}(\mathbf{x})\left\|\mathbf{P}_{a}(\mathbf{x}) \mathbf{y}\right\|^{2} \\
\pi_{4}(\mathbf{x}, \mathbf{y}, \mathbf{r}) & =\frac{1}{2}\left\|\mathbf{J}_{a}^{T}(\mathbf{x}) \mathbf{r}_{a}^{\prime}(\mathbf{x})-\mathbf{P}_{a}(\mathbf{x}) \mathbf{y}\right\|^{2}
\end{aligned}
$$

Showing the properness of $\pi_{3}^{\times}$and $\pi_{4}$ is similar to that of $\boldsymbol{\pi}_{1}^{\times}$. We can easily derive the $\boldsymbol{\pi}_{3}^{\times}$-PIK solution as

$$
\mathbf{u}=\mathbf{R}^{-1} \sum_{a=1}^{l} \hat{\mathbf{J}}_{a}^{T} \mathbf{C}_{a a}^{\times} \mathbf{r}_{a}^{\prime}=\mathbf{R}^{-1} \hat{\mathbf{J}}^{T} \mathbf{C}_{D}^{\otimes} \mathbf{r}^{\prime}
$$

and the $\pi_{4}$-PIK solution as

$$
\mathbf{u}=\mathbf{R}^{-1} \sum_{a=1}^{l} \hat{\mathbf{J}}_{a}^{T} \mathbf{C}_{a a}^{T} \mathbf{r}_{a}^{\prime}=\mathbf{R}^{-1} \hat{\mathbf{J}}^{T} \mathbf{C}_{D}^{T} \mathbf{r}^{\prime}
$$

where $\mathbf{C}_{D}^{\otimes}=\operatorname{diag}\left(\mathbf{C}_{11}^{\times}, \ldots, \mathbf{C}_{l l}^{\times}\right)$.

Remark 2: We can simplify the $\mathrm{QR}$ decomposition $\mathbf{J}^{T}=$ $\hat{\mathbf{J}}_{e}^{T} \mathbf{C}_{e}^{T}=\hat{\mathbf{J}}^{T} \mathbf{C}^{T}$ given by Lemma 1 when formulating the PIK solutions introduced in this section. Observe that every 
solution starts by $\mathbf{R}^{-1} \hat{\mathbf{J}}^{T} \mathbf{C}_{D}^{T}$ and the latter part does not include $\hat{\mathbf{J}}$. Construct $\tilde{\mathbf{J}}=\left[\begin{array}{lll}\tilde{\mathbf{j}}_{1}^{T} & \cdots & \tilde{\mathbf{j}}_{m}^{T}\end{array}\right]^{T}: X \rightarrow \mathbb{R}^{m \times n}$ by letting $\tilde{\mathbf{j}}_{a}(\mathbf{x})=\hat{\mathbf{j}}_{a}(\mathbf{x})$ if $c_{a a}(\mathbf{x})>0$ and $\tilde{\mathbf{j}}_{a}(\mathbf{x})=\mathbf{0}$ if $c_{a a}(\mathbf{x})=0$ for all $(a, \mathbf{x}) \in \overline{1, m} \times X$. Since $c_{a b}(\mathbf{x})=0$ for all $a \in \overline{1, m}$ if $c_{b b}(\mathbf{x})=0$, we have $\mathbf{J}^{T}=\hat{\mathbf{J}}^{T} \mathbf{C}^{T}=\tilde{\mathbf{J}}^{T} \mathbf{C}^{T}$ and $\hat{\mathbf{J}}^{T} \mathbf{C}_{D}^{T}=\tilde{\mathbf{J}}^{T} \mathbf{C}_{D}^{T}$. The decomposition $\mathbf{J}^{T}=\tilde{\mathbf{J}}^{T} \mathbf{C}^{T}$ can be obtained by running the algorithm in the proof of Lemma 1 where $\tilde{\mathbf{J}}^{T}=\tilde{\mathbf{Q}}_{r}$ and $\mathbf{C}^{T}=\mathbf{R}_{r}$. So, we do not need to find orthonormal basis of $\mathcal{N}(\mathbf{J}(\mathbf{x}))$ in order to formulate the PIK solutions introduced in this section.

It would be interesting to see how those PIK solutions work. We provide numerical simulations with a 7-DOF manipulator, KUKA LWR, whose first priority task is to move the endeffector position $\mathbf{f}_{1}(\mathbf{q}) \in \mathbb{R}^{3}$, second priority task is to adjust the end-effector orientation $\mathbf{f}_{2}(\mathbf{q}) \in \mathbb{R}^{3}$ represented by the rotation vector, and third priority task is to avoid a stationary obstacle. The obstacle avoidance is implemented based on [5] in which a repulsive velocity is generated to make the closest point on the manipulator move away from the obstacle. We choose the initial configuration $\mathbf{q}_{0} \in \mathbb{R}^{7}$ satisfying $\operatorname{rank}\left(\mathbf{F}_{q}\left(\mathbf{q}_{0}\right)\right)=7$; let $\mathbf{p}_{a} \in \mathbb{R}^{3}$ for $a \in \overline{1,2}$ sufficiently close to $\mathbf{f}_{a}\left(\mathbf{q}_{0}\right)$; and design the $a$-th reference for $a \in \overline{1,2}$ as $\mathbf{r}_{a}(\mathbf{q})=10\left(\mathbf{p}_{a}-\mathbf{f}_{a}(\mathbf{q})\right)$. The preconditioner function $\mathbf{R}$ is determined based on [37] with $\delta=0.2$. We generate the joint trajectory $\mathbf{q}(t)$ from $\boldsymbol{\pi}_{1}^{+-}, \boldsymbol{\pi}_{2}^{+}-, \boldsymbol{\pi}_{3}^{+}$-, and $\boldsymbol{\pi}_{4}$-PIK solutions and observe if the norms of task errors $\left\|\mathbf{e}_{a}(t)\right\|=\left\|\mathbf{p}_{a}-\mathbf{f}_{a}(\mathbf{q}(t))\right\|$ for $a \in \overline{1,2}$ converge to zero, if the minimum distance $d(t)$ between the manipulator and the obstacle increases, and if the norms of residuals $\left\|\mathbf{e}_{a}^{\text {res }}(t)\right\|=\left\|\mathbf{r}_{a}(\mathbf{q}(t))-\mathbf{F}_{a}(\mathbf{q}(t)) \dot{\mathbf{q}}(t)\right\|$ for $a \in \overline{1,3}$ converge to zero as $t \rightarrow \infty$.

The simulation results are shown in Figure 1. The joint trajectory satisfying $\dot{\mathbf{q}}(t)=\mathbf{u}(\mathbf{q}(t))$ is found numerically by using the Euler method with the fixed time step of $1 \mathrm{~ms}$. It is clear that all PIK solutions give the desired behavior but the convergence speed is high for $\boldsymbol{\pi}_{1}^{+}$- and $\boldsymbol{\pi}_{3}^{+}$-PIK solutions, medium for the $\pi_{2}^{+}$-PIK solution, and low for the $\pi_{4}$-PIK solution. Figure 2 shows that the joint velocity of the $\pi_{4}$-PIK solution is much lower than others; it could be the reason of the low convergence speed. However, the $\pi_{4}$-PIK solution does not diverge near singularity because it does not contain the inverse of a matrix, while the others should be replaced to the damped version.

\section{CONCLUSION}

We showed that how the PIK problem can be generalized to the multi-objective optimization with the lexicographical ordering. We found three properties (O1) to (O3) for a vectorvalued objective function to be proper for the PIK problem by reasoning that a PIK solution should have dependence $(\mathrm{O} 1)$, uniqueness $(\mathrm{O} 2)$, and representation $(\mathrm{O} 3)$ properties to preserve priority relations between tasks. As a result, we could define the set of all PIK solutions from the set of all proper objective functions by the mapping defined as the optimal solution of the multi-objective optimization with the lexicographical ordering. We showed that our generalization discards some trivial solutions and includes Nakamura's and (weighted) Chiaverini's solutions with and without damping.

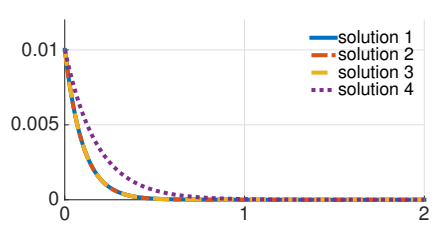

(a) $\left\|\mathbf{e}_{1}(t)\right\|$

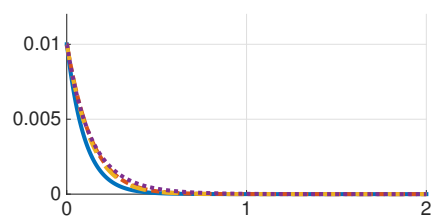

(c) $\left\|\mathbf{e}_{2}(t)\right\|$

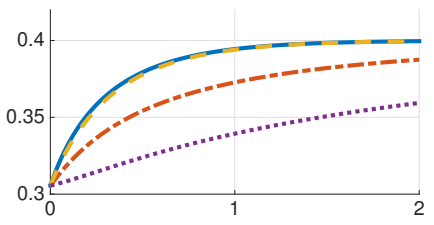

(e) $d(t)$

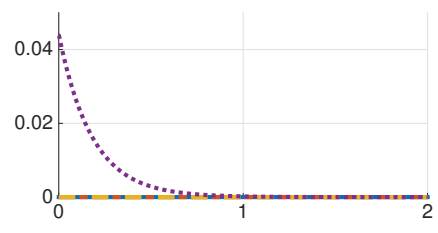

(b) $\left\|\mathbf{e}_{1}^{\mathrm{res}}(t)\right\|$

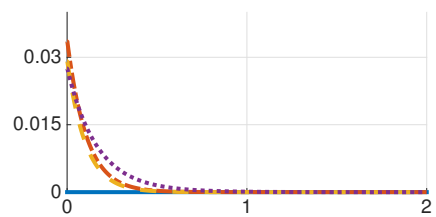

(d) $\left\|\mathbf{e}_{2}^{\text {res }}(t)\right\|$

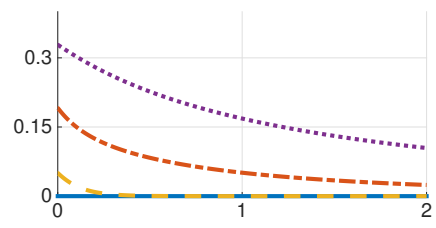

(f) $\left|e_{3}^{\text {res }}(t)\right|$
Fig. 1. Norms of task errors, the minimum distance, and norms of residuals are plotted with respect to the time $t[\mathrm{~s}]$.

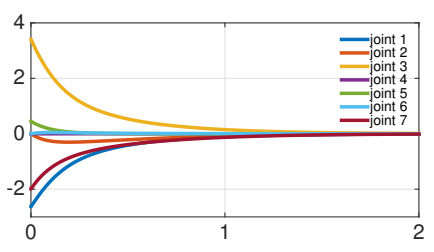

(a) $\pi_{1}^{+}$-PIK solution

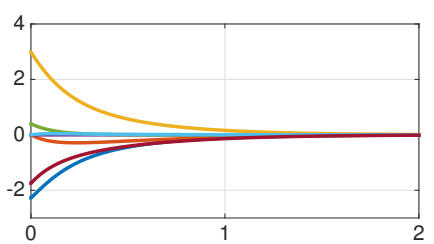

(c) $\pi_{3}^{+}$-PIK solution

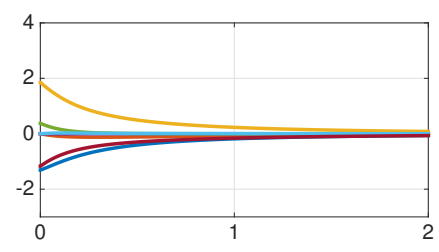

(b) $\pi_{2}^{+}$-PIK solution

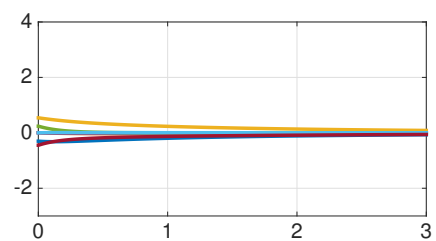

(d) $\pi_{4}$-PIK solution
Fig. 2. Joint velocities $\dot{q}_{i}(t)[\mathrm{rad} / \mathrm{s}]$ for $i \in \overline{1,7}$ are plotted with respect to the time $t[\mathrm{~s}]$.

We also furnished some other PIK solutions that have not been formulated so far and compared their properties by a simulation of a 7-DOF manipulator, KUKA LWR.

Our generalization opens a possibility to study theoretical properties of the PIK problem. For example, one can find an answer of the question "Can we always find a smooth PIK solution given smooth Jacobians and references?" from [38]. Also, our generalization provides an intuitive and systematic way to find new PIK solutions; it would be easier to design proper objective functions than to find PIK solutions directly.

\section{APPENDIX}

Run the next algorithm.

1: $\mathbf{V}=\left[\begin{array}{lll}\mathbf{v}_{1} & \cdots & \mathbf{v}_{m}\end{array}\right] \leftarrow \mathbf{J}^{T}$

2: $\tilde{\mathbf{Q}}_{r}=\left[\begin{array}{lll}\mathbf{q}_{1} & \cdots & \mathbf{q}_{m}\end{array}\right] \leftarrow \mathbf{0} \in \mathbb{R}^{n \times m}$

3: $\mathbf{R}_{r}=\left[r_{i j}\right] \leftarrow \mathbf{0} \in \mathbb{R}^{m \times m}$ 


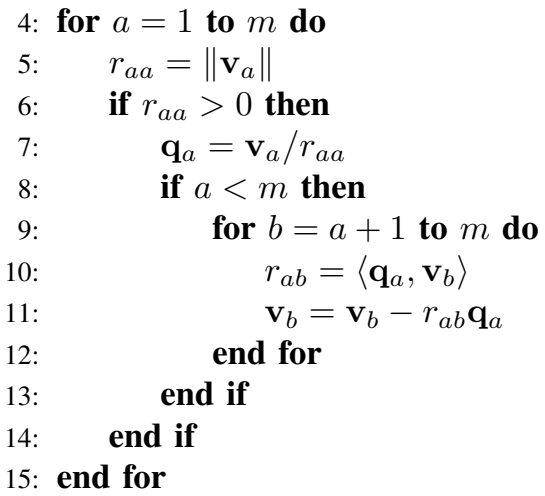

Then, we get the decomposition $\mathbf{J}^{T}=\tilde{\mathbf{Q}}_{r} \mathbf{R}_{r}$ where $\mathbf{R}_{r}$ is upper triangular; $r_{a a} \geq 0$ for $a \in \overline{1, m} ; r_{a b}=0$ for $b \in \overline{1, m}$ if $r_{a a}=0 ; \mathbf{q}_{a}=\mathbf{0}$ if and only if $r_{a a}=0$; and nonzero columns of $\tilde{\mathbf{Q}}_{r}$ are orthonormal. Define $\tilde{\mathbf{Q}} \in \mathbb{R}^{n \times n}$ and $\mathbf{R} \in \mathbb{R}^{n \times m}$ as $\tilde{\mathbf{Q}}=\tilde{\mathbf{Q}}_{r}$ and $\mathbf{R}=\mathbf{R}_{r}$ if $m=n$ and $\tilde{\mathbf{Q}}=\left[\begin{array}{ll}\tilde{\mathbf{Q}}_{r} & \mathbf{0}\end{array}\right]$ and $\mathbf{R}=\left[\begin{array}{ll}\mathbf{R}_{r}^{T} & \mathbf{0}\end{array}\right]^{T}$ if $m<n$. There are only $r=\operatorname{rank}(\mathbf{J})$ nonzero columns in $\tilde{\mathbf{Q}}$. Let $\left\{\mathbf{p}_{1}, \ldots, \mathbf{p}_{n-r}\right\} \subset$ $\mathbb{R}^{n}$ be an orthonormal basis of $\mathcal{N}(\mathbf{J})$. Construct $\mathbf{Q} \in \mathbb{R}^{n \times n}$ by replacing zero columns of $\tilde{\mathbf{Q}}$ with $\mathbf{p}_{1}, \ldots, \mathbf{p}_{n-r}$. Since $\mathcal{R}(\tilde{\mathbf{Q}})=\mathcal{R}\left(\mathbf{J}^{T}\right) \perp \mathcal{N}(\mathbf{J}), \mathbf{Q}$ is orthogonal and we get the full QR decomposition $\mathbf{J}^{T}=\mathbf{Q R}=\hat{\mathbf{J}}_{e}^{T} \mathbf{C}_{e}^{T}$.

\section{REFERENCES}

[1] N. Mansard, O. Khatib, and A. Kheddar, "A Unified Approach to Integrate Unilateral Constraints in the Stack of Tasks," IEEE Transactions on Robotics, vol. 25, no. 3, pp. 670-685, 2009.

[2] A. Escande, N. Mansard, and P.-B. Wieber, "Fast resolution of hierarchized inverse kinematics with inequality constraints," in IEEE International Conference on Robotics and Automation, 2010, pp. 3733 3738.

[3] O. Kanoun, "Real-Time Prioritized Kinematic Control under Inequality Constraints for Redundant Manipulators," in Robotics: Science and Systems, 2012, pp. 145-152.

[4] A. Escande, N. Mansard, and P. B. Wieber, "Hierarchical quadratic programming: Fast online humanoid-robot motion generation," International Journal of Robotics Research, vol. 33, no. 7, pp. 1006-1028, 2014.

[5] L. Žlajpah and B. Nemec, "Kinematic control algorithms for on-line obstacle avoidance for redundant manipulators," in IEEE/RSJ International Conference on Intelligent Robots and Systems, 2002, pp. 1898-1903.

[6] F. Keith, P.-b. Wieber, N. Mansard, and A. Kheddar, "Analysis of the Discontinuities in Prioritized Tasks-Space Control Under Discreet Task Scheduling Operations," in IEEE/RSJ International Conference on Intelligent Robots and Systems, 2011, pp. 3887-3892.

[7] J. Lee, N. Mansard, and J. Park, "Intermediate Desired Value Approach for Task Transition of Robots in Kinematic Control," IEEE Transactions on Robotics, vol. 28, no. 6, pp. 1260-1277, 2012.

[8] T. Petrič and L. Žlajpah, "Smooth continuous transition between tasks on a kinematic control level: Obstacle avoidance as a control problem," Robotics and Autonomous Systems, vol. 61, no. 9, pp. 948-959, 2013.

[9] S.-i. An and D. Lee, "Prioritized Inverse Kinematics with Multiple Task Definitions," in IEEE International Conference on Robotics and Automation, 2015, pp. 1423-1430.

[10] O. Khatib, "A Unified Approach for Motion and Force Control of Robot Manipulators: The Opeartional Space Formulation,” IEEE Journal on Robotics and Automation, vol. 3, no. 1, pp. 43-53, 1987.

[11] P. Hsu, J. Mauser, and S. Sastry, "Dynamic control of redundant manipulators," Journal of Robotic Systems, vol. 6, no. 2, pp. 133-148, 1989.

[12] P. H. Chang and J. W. Jeong, "Enhanced Operational Space Formulation for Multiple Tasks by Using Time-Delay Estimation," IEEE Transactions on Robotics, vol. 28, no. 4, pp. 773-786, 2012.

[13] H. Sadeghian, L. Villani, M. Keshmiri, and B. Siciliano, "Dynamic multi-priority control in redundant robotic systems," Robotica, vol. 31, no. 7 , pp. 1155-1167, 2013.
[14] H. Sadeghian, L. Villani, M. Keshmiri, and B. Siciliano, "Task-space control of robot manipulators with null-space compliance," IEEE Transactions on Robotics, vol. 30, no. 2, pp. 493-506, 2014.

[15] C. Ott, A. Dietrich, and A. Albu-Schäffer, "Prioritized multi-task compliance control of redundant manipulators," Automatica, vol. 53, pp. 416-423, 2015.

[16] Y. Nakamura and H. Hanafusa, "Optimal Redundancy Control of Robot Manipulators," The International Journal of Robotics Research, vol. 6, no. 1 , pp. 32-42, 1987.

[17] C. Schuetz, T. Buschmann, J. Baur, J. Pfaff, and H. Ulbrich, "Predictive Online Inverse Kinematics for Redundant Manipulators," in IEEE International Conference on Robotics and Automation, 2014, pp. 5056-5061.

[18] A. Del Prete, F. Romano, L. Natale, G. Metta, G. Sandini, and F. Nori, "Prioritized optimal control," in IEEE International Conference on Robotics and Automation, 2014, pp. 2540-2545.

[19] F. Romano, A. Prete Del, N. Mansard, and F. Nori, "Prioritized Optimal Control : a Hierarchical Differential Dynamic Programming approach," in IEEE International Conference on Robotics and Automation, 2015, pp. 3590-3595.

[20] M. Geisert, A. Del Prete, N. Mansard, F. Romano, and F. Nori, "Regularized hierarchical differential dynamic programming," IEEE Transactions on Robotics, vol. 33, no. 4, pp. 819-833, 2017.

[21] M. Saveriano, S.-i. An, and D. Lee, "Incremental Kinesthetic Teaching of End-Effector and Null-Space Motion Primitives," in IEEE International Conference on Robotics and Automation, 2015, pp. 3570-3575.

[22] V. Modugno, G. Neumann, E. Rueckert, G. Oriolo, J. Peters, and S. Ivaldi, "Learning soft task priorities for control of redundant robots," in IEEE International Conference on Robotics and Automation, 2016, pp. 221-226.

[23] S. Calinon, "A tutorial on task-parameterized movement learning and retrieval," Intelligent Service Robotics, vol. 9, no. 1, pp. 1-29, 2016.

[24] S. Calinon, "Robot Learning with Task-Parameterized Generative Models," Robotics Research, pp. 111-126, 2018.

[25] J. Silverio, S. Calinon, L. Rozo, and D. G. Caldwell, "Learning Task Priorities From Demonstrations," IEEE Transactions on Robotics, vol. 53, no. 1, pp. 78-94, 2019.

[26] N. J. Dehio, "Prioritized Multi-Objective Robot Control," Doctoral dissertation, Technische Universität Braunschweig, 2018.

[27] K. Bouyarmane and A. Kheddar, "On weight-prioritized multitask control of humanoid robots," IEEE Transactions on Automatic Control, vol. 63, no. 6, pp. 1632-1647, 2018.

[28] Y. Nakamura, H. Hanafusa, and T. Yoshikawa, "Task-Priority Based Redundancy Control of Robot Manipulators," The International Journal of Robotics Research, vol. 6, no. 2, pp. 3-15, 1987.

[29] A. A. Maciejewski and C. A. Klein, "Obstacle Avoidance for Kinematically Redundant Manipulators in Dynamically Varying Environments," The International Journal of Robotics Research, vol. 4, no. 3, pp. 109$117,1985$.

[30] B. Siciliano and J.-J. E. Slotine, "A General Framework for Managing Multiple Tasks in Highly Redundant Robotic Systems,' in International Conference on Advanced Robotics, 1991, pp. 1211-1216.

[31] S. Chiaverini, "Singularity-Robust Task-Priority Redundancy Resolution for Real-Time Kinematic Control of Robot Manipulators," IEEE Transactions on Robotics and Automation, vol. 13, no. 3, pp. 398-410, 1997.

[32] P. Baerlocher and R. Boulic, "Task-Priority Formulations for the Kinematic Control of Highly Redundant Articulated Structures," in IEEE/RSJ International Conference on Intelligent Robots and Systems, 1998, pp. 323-329.

[33] J. Park, Y. Choi, W. K. Chung, and Y. Youm, "Multiple Tasks Kinematics Using Weighted Pseudo-Inverse for Kinematically Redundant Manipulators," in IEEE International Conference on Robotics and Automation, 2001, pp. 4041-4047.

[34] Y. Choi, Y. Oh, S. R. Oh, J. Park, and W. K. Chung, "Multiple tasks manipulation for a robotic manipulator," Advanced Robotics, vol. 18, no. 6, pp. 637-653, 2004.

[35] F. H. Clarke, Y. S. Ledyaev, R. J. Stern, and P. R. Wolenski, Nonsmooth analysis and control theory. Springer, 2008.

[36] L. N. Trefethen and B. David III., Numerical linear algebra. Siam, 1997.

[37] S.-i. An and D. Lee, "Prioritized Inverse Kinematics using QR and Cholesky Decompositions," in IEEE International Conference on Robotics and Automation, 2014, pp. 5062-5069.

[38] S.-i. An and D. Lee, "Prioritized Inverse Kinematics : Nonsmoothness , Trajectory Existence , Task Convergence , Stability," arXiv:1905.03416v1 [cs.SY], 2019. 